\title{
Chondroitinase Combined with Rehabilitation Promotes Recovery of Forelimb Function in Rats with Chronic Spinal Cord Injury
}

\author{
Difei Wang, ${ }^{1}$ Ronaldo M. Ichiyama, ${ }^{2}$ Rongrong Zhao, ${ }^{1}$ Melissa R. Andrews, ${ }^{1}$ and James W. Fawcett ${ }^{1}$ \\ ${ }^{1}$ Cambridge University Centre for Brain Repair, Cambridge CB2 OPY, United Kingdom, and ${ }^{2}$ Institute of Membrane and Systems Biology, University of \\ Leeds, Leeds LS2 9JT, United Kingdom
}

Chondroitinase $\mathrm{ABC}(\mathrm{ChABC})$ in combination with rehabilitation has been shown to promote functional recovery in acute spinal cord injury. For clinical use, the optimal treatment window is concurrent with the beginning of rehabilitation, usually $2-4$ weeks after injury. We show that $\mathrm{ChABC}$ is effective when given 4 weeks after injury combined with rehabilitation. After $\mathrm{C} 4$ dorsal spinal cord injury, rats received no treatment for 4 weeks. They then received either ChABC or penicillinase control treatment followed by hour-long daily rehabilitation specific for skilled paw reaching. Animals that received both ChABC and task-specific rehabilitation showed the greatest recovery in skilled paw reaching, approaching similar levels to animals that were treated at the time of injury. There was also a modest increase in skilled paw reaching ability in animals receiving task-specific rehabilitation alone. Animals treated with ChABC and taskspecific rehabilitation also showed improvement in ladder and beam walking. ChABC increased sprouting of the corticospinal tract, and these sprouts had more vGlut1 ${ }^{+\mathrm{ve}}$ presynaptic boutons than controls. Animals that received rehabilitation showed an increase in perineuronal net number and staining intensity. Our results indicate that $\mathrm{ChABC}$ treatment opens a window of opportunity in chronic spinal cord lesions, allowing rehabilitation to improve functional recovery.

\section{Introduction}

Many compounds stimulate axon regeneration or plasticity in the injured spinal cord. In most experiments, these have been applied around the time of injury. For human patients, however, while it is possible in selected cases to apply a regenerative treatment soon after injury, this timing is far from optimal. Many patients are either too ill, or it is not possible to fit another intervention into the clinical plan. The functional outcome soon after injury is also very uncertain, and this variability makes it necessary to include large patient numbers in clinical trials (Fawcett et al., 2007; Lammertse et al., 2007; Steeves et al., 2007; Tuszynski et al., 2007). The optimal time to initiate treatment would be at the same time as rehabilitation (rehab) is begun, usually 2-4 weeks after injury. So far, few potential therapeutics have been tested in animal models of chronic spinal cord injury (SCI). Limited functional recovery has been seen using olfactory ensheathing cells (Keyvan-Fouladi et al., 2003, 2005); neurotrophins with bone marrow stroma cells (Kadoya et al., 2009), with fetal transplants

\footnotetext{
Received Feb. 23, 2011; revised May 7, 2011; accepted May 10, 2011.

Author contributions:D.W. and J.W.F. designed research;D.W. performed research; D.W., R.M.I., R.Z., and M.R.A. contributed unpublished reagents/analytic tools; D.W. analyzed data; D.W. and J.W.F. wrote the paper.

This work was supported by grants from the Medical Research Council, The Christopher and Dana Reeve Foundation, The European Union Framework 7 projects Spinal Cord Repair and Plasticise, the NIHR Cambridge Biomedical Research Centre, The John and Lucille van Geest Foundation, and the Henry Smith Charity. We thank Jessica Kwok and Guillermo García-Alías for their helpful advice; David Story for technical support; and Christopher Aylett for discussion and critical reading of this manuscript.

Correspondence should be addressed to James W. Fawcett, Cambridge University Centre for Brain Repair, Robinson Way, Cambridge CB2 OPY, UK. E-mail: jf108@cam.ac.uk.

DOI:10.1523/JNEUROSCI.0983-11.2011

Copyright $\odot 2011$ the authors $\quad 0270-6474 / 11 / 319332-13 \$ 15.00 / 0$
}

(Coumans et al., 2001; Zurita et al., 2001) or neurotrophinsecreting fibroblasts (Shumsky et al., 2003; Tobias et al., 2003); grafts of intercostal nerves (Fraidakis et al., 2004) and preinjured sural nerve (Feng et al., 2008); and chondroitinase in combination with peripheral nerve grafts (Tom et al., 2009a) or adult neural stem/progenitor cells (Karimi-Abdolrezaee et al., 2006, 2010).

The bacterial enzyme chondroitinase ABC (ChABC), which digests extracellular chondroitin sulfate proteoglycans (CSPGs), has been shown to enhance axonal regeneration both in vitro and in vivo (Zuo et al., 1998; Yick et al., 2000, 2003; Bradbury et al., 2002; Moon et al., 2002; Tester and Howland, 2008). ChABC also reactivates plasticity in the adult CNS (Pizzorusso et al., 2002; Galtrey et al., 2007), its main target being digestion of perineuronal nets (Carulli et al., 2010). Its positive effects on recovery of function after SCI are probably mainly due to enhancement of plasticity rather than long-distance axonal regeneration (GarcíaAlías et al., 2009). In clinical practice, rehabilitation is used to drive the limited plasticity of the adult CNS, and has various effects on cellular and molecular functions (Vaynman and Gomez-Pinilla, 2005; Cotman et al., 2007; Ying et al., 2008). Recent results from our laboratory have shown that in an acute treatment model, ChABC-enhanced plasticity greatly increases the efficacy of specific rehabilitation. The present study is aimed at assessing the effectiveness of ChABC treatment on chronic SCI. ChABC paired with specific rehabilitation was delivered to animals 4 weeks after SCI. The method and timing of treatment and its combination with rehabilitation closely parallel the way in which a regenerative treatment might be delivered to human 
patients. There was significant functional recovery in skilled paw reaching and other forelimb motor functions accompanied by increased spouting and synapse formation by corticospinal and serotonergic axons. Our results suggest that delayed ChABC treatment would be effective if given to patients as they start rehabilitation after SCI.

\section{Materials and Methods}

All procedures were performed in compliance with the UK Animals (Scientific Procedures) Act 1986 and institutional guidelines.

\section{Spinal cord injury}

Male Lister hooded rats $(150-200 \mathrm{~g})$ were deeply anesthetized with 1-2\% isoflurane in a mixture of $25 \%$ nitrous oxide, $50 \%$ oxygen and air. An SCI was performed as previously described by (García-Alías et al., 2009). Briefly, a C4 laminectomy was performed exposing the spinal cord beneath. A cut was made with the tips of sharpened fine forceps (Dumont no. 5) inserted $2 \mathrm{~mm}$ in depth into the spinal cord parenchyma spanning the gap between the dorsal root entries and down to the spinal canal. The injury included the descending dorsal corticospinal tracts (CSTs) and the ascending sensory dorsal columns.

\section{Drug delivery to chronic SCI}

One month after the SCI, the animals were anesthetized as before and the $\mathrm{C} 4$ vertebrum re-exposed. The overlying scar tissue was carefully dissected away to expose the cord $2 \mathrm{~mm}$ above and below the lesion. Laminectomy of the $\mathrm{C} 3$ and $\mathrm{C} 5$ vertebra was performed if necessary to allow a clear field for injection. Two $1 \mu \mathrm{l}$ injections $2 \mathrm{~mm}$ above and below the lesion site of either ChABC (100 U/ml, protease free, Seikagaku) or control bacterial enzyme [penicillinase (Pen), $2 \mathrm{mg} / \mathrm{ml}$ matched for protein content, Sigma] were made through a pulled glass capillary with a tip diameter of $\sim 20 \mu \mathrm{m}$ (Harvard Instruments) at the rate of $6 \mu \mathrm{l} / \mathrm{h}$. A 32 gauge catheter (ReCathCo) was inserted intrathecally through the opening of the cisterna magna, with the tip lying on top of the injury. The tube was fixed onto the skull and externalized over the head between the eyes. The animals received a total of five injections of ChABC through the cannula ( $3 \mu \mathrm{l}, 100 \mathrm{U} / \mathrm{ml}$ per injection, Acorda Therapeutics) or Pen ( $3 \mu \mathrm{l}$, $100 \mathrm{U} / \mathrm{ml}$ per injection), once every $2 \mathrm{~d}$ following surgery. This is the same protocol of administration used in our previous studies (GarcíaAlías et al., 2008, 2009). Previous work from our group has shown that active $\mathrm{ChABC}$ can be retrieved for up to 3 weeks after intraparenchymal injections (Lin et al., 2008, Hyatt et al., 2010).

\section{Experimental groups}

The animals were divided into four experimental groups. All the animals received the cervical SCI, and chondroitinase or penicillinase infusion with or without task-specific rehabilitation as follows: ChABC rehab group $(n=13)$; Pen rehab group $(n=11)$; ChABC no rehab group $(n=$ $10)$; or Pen no rehab group $(n=10)$. Because of the large number of animals, the experiment was performed in two stages. Each stage contained control animals that received $\mathrm{ChABC}$ or Pen without rehabilitation. There was no difference in behavioral recovery between the groups in the two stages.

\section{Task-specific rehabilitation}

One week after the injection of the treatment into the spinal cord, one group of the animals receiving $\mathrm{ChABC}$ and one group of the animals receiving penicillinase were started on task-specific rehabilitation. Briefly, the animals were placed in the Montoya-type staircase reaching cages (Montoya et al., 1991; Galtrey et al., 2007). The device contains a staircase of seven small wells $8 \mathrm{~mm}$ deep at increasing distance from the rat on either side of a central divide. The wells were overfilled with a variety of seeds, and animals were encouraged to grasp for the seeds for 30 min twice daily.

\section{Behavioral assessment}

Staircase reaching task. For 3 weeks before injury, animals were trained to grasp and eat sugar pellets from the Montoya-type staircase device, the same as the one used for task-specific rehabilitation, except two sugar pellets were placed in each well instead of seeds. Thirty percent of the animals had failed to achieve retrieval of $\geq 16$ pellets and were excluded from the study at this stage. For assessment, the rats were given $15 \mathrm{~min}$ to remove and eat as many sugar pellets as possible. The number of pellets displaced, eaten, or dropped and the maximum level reached was recorded (Montoya et al., 1991). The accuracy was calculated as the percentage of sugar pellets displaced that were eaten.

Horizontal bar and beam walking. Animals were videotaped walking along a horizontal ladder $120 \mathrm{~cm}$ long with unevenly spaced bars. The total number of forepaw steps made and the number of slips were recorded. The animals were also videotaped walking along a beam of decreasing width from 5 to $2 \mathrm{~cm}$, and number of forelimb slips was recorded.

Gait analysis. The forepaws and hindpaws of the animals were inked with paint of different colors, and footprints were made on paper as the animals walked along a $120-\mathrm{cm}$-long runway toward a reward. A series of at least three sequential steps was used to determine the mean values of hindlimb base of support and hindlimb and forelimb stride length. The forepaw and hindpaw stride lengths were determined by measuring the distance between two consecutive prints and were averaged over three strides. The base of support was determined by measuring the distance between two consecutive right and left hindpaw prints (Metz and Schwab, 2004).

\section{Sensory testing}

Sensory testing was performed at the end of the behavioral assessment. For testing, rats were placed in Plexiglas containers with a wire mesh floor (pressure test) or a Plexiglas floor (hot-plate test), and acclimatized to the testing chamber for $10 \mathrm{~min}$. Fine touch and mechanical hyperalgesia were assessed with a fine probe and an electronic anesthesiometer (model 1601C, Life Science Instruments). The probe was gently applied to the glabrous foot pad of the left forepaw, and pressure was increased until the rat withdrew its paw. If the rat did not withdraw its paw with a force of $150 \mathrm{~g}$, the test was terminated. The pressure $(\mathrm{g})$ reached when rat withdrew its paw was recorded with a force transducer. The trials were performed on the left and right paw alternatively. Five trials were performed on each paw during the testing period with the lowest and highest recordings discarded. Thermal hyperalgesia were measured using a hotplate device. A movable infrared light source (Ugo Basile) was placed under the center of the foot pad of the forepaw and used to generate heat on a localized spot. The time (in seconds) at which the animal withdrew its paw after the stimulus began was recorded. The test was terminated if the time exceeded $20 \mathrm{~s}$. Three trials were performed per testing session.

\section{Corticospinal axon tracing}

At the end of the behavioral evaluation, the animals were anesthetized with isoflurane and placed in a stereotaxic frame. Biotinylated dextran amine (BDA) (10\% w/v, MW 10,000, Invitrogen) was injected stereotaxically at a depth of $1.5 \mathrm{~mm}$ to the following coordinates: anteroposterior $(\mathrm{AP})+0.5$, mediolateral $(\mathrm{ML}) \pm 2 ; \mathrm{AP}-0.5, \mathrm{ML} \pm 3 ; \mathrm{AP}-0.5, \mathrm{ML}$ \pm 1.8 (Hagg et al., 2005) over the sensorimotor cortex. One microliter was injected at each site.

\section{Histology}

Two weeks after tracer injection, the animals were killed and transcardially perfused with PBS followed by $4 \%$ paraformaldehyde in $0.1 \mathrm{~m}$ phosphate buffer. Brains and the $\mathrm{C} 1, \mathrm{C} 3-\mathrm{C} 5, \mathrm{~T} 2$ spinal cord segments were removed and postfixed in $4 \%$ paraformaldehyde solution at $4^{\circ} \mathrm{C}$ overnight, followed by $30 \%$ sucrose for $72 \mathrm{~h}$. Tissue was frozen in OCT mounting medium, and transverse $30 \mu \mathrm{m}$ sections of the brain and longitudinal sections of the $\mathrm{C} 3-\mathrm{C} 5$ spinal segments were cut with a cryostat and processed for immunohistochemistry.

To verify the injury to the corticospinal tract, transverse sections of the $\mathrm{C} 1$ and T2 spinal segments as well as transverse sections of the lesion site were immunostained for PKC $\gamma$ (rabbit polyclonal anti-PKC $\gamma, 1: 1000$, Millipore Bioscience Research Reagents). For visualization of BDAlabeled fibers, one in five longitudinal sections was processed by the avidin-biotin amplification method with conjugated peroxidase (Vectastain ABC Elite Kit, Vector Laboratories) and visualized with diaminobenzidine $(\mathrm{DAB})$ and $\mathrm{NiCl}_{2}$.

For visualization of 5-HT-positive $\left(5-\mathrm{HT}^{+\mathrm{ve}}\right)$ fibers, 1 in 10 longitudinal sections was immunostained for 5-HT (goat anti-5-HT, 1:1000, 
Immunostar). For visualization of calcitonin gene-related peptide-positive $\left(\mathrm{CGRP}^{+\mathrm{ve}}\right) \mathrm{fi}^{\mathrm{f}}$ bers, 1 in 10 longitudinal sections were immunostained for CGRP (rabbit anti-CGRP, 1:1000, Sigma). For visualization of colocalization of vesicular glutamate transporter 1 (vGlut1) and BDA, one in five longitudinal sections was incubated with guinea pig antivGlut1 antibody (1:1000, Millipore Bioscience Research Reagents), then Cy3-conjugated donkey anti-guinea pig antibody (1:500) and streptavidin conjugated to Alexa Fluor 488 (1: 500, Invitrogen).

For visualization of CSPG GAG digestion, six animals with chronic spinal cord injuries (1 month old), having received either ChABC $(n=4)$ or Pen $(n=2)$ injections and intrathe$\mathrm{cal}$ infusion were perfused the day after the last infusion. A spinal block from C2 to C6 was removed and processed. Sections were immunostained with anti-chondroitin sulfate DDi-OS monoclonal antibodies (1: 200, Seikagaku) and mouse monoclonal anti-neurocan (1:5, Developmental Studies Hybridoma Bank), then goat anti-mouse biotinylated antibodies (1:500, Vector Laboratories), amplified using the avidin-biotin method with peroxide as a substrate (Vectastain ABC Elite Kit, Vector Laboratories), and then stained with DAB.

For histological analysis of the chronic lesion, six animals received a cervical SCI. Three were then perfused $4 \mathrm{~d}$ after injury, and three were perfused 1 month after the injury. In addition, three normal rats with sham injury were also perfused as negative controls. A spinal block from $\mathrm{C} 2$ to $\mathrm{C} 6$ was removed as before and processed. Horizontal sections were examined for laminin, GFAP, neurocan, OX42, and MBP immunoreactivity. The antibodies used were as follows: rabbit anti-laminin (1:1000, Chemicon); rabbit anti-GFAP (1:1000, DAKO); mouse monoclonal anti-neurocan (1:5, Developmental Studies Hybridoma Bank); mouse monoclonal anti-OX42 (1:200, Sigma); and rabbit anti-MBP (1:500, Abcam). All Alexa Fluor-tagged secondary antibodies were from Invitrogen. To evaluate antibody specificity, some sections were processed as described, but primary antibodies were not added.

For histological analysis of the extracellular matrix (ECM) after training, three animals received task-specific training from 4 weeks after injury, whereas three further animals did not receive any training after injury. These six animals were killed and transcardially perfused 18 weeks after injury. The spinal block from C2 to C6 was removed and processed as before. Horizontal sections were examined for Link Protein (Crtll, 1:100, Santa Cruz Biotechnology), lectin Wisteria fluribunda (WFA) $1: 150$, Sigma), and aggrecan (1:100, Millipore) immunoreactivity, with each section coimmunostained with NeuN (1:200, Millipore Bioscience Research Reagents) for visualization of neurons. All Alexa Fluor-tagged secondary antibodies were from Invitrogen. To evaluate antibody specificity, some sections were processed as described, but primary antibodies were not added.

\section{Lesion size and corticospinal axon quantification}

Corticospinal axons were quantified from the horizontal sections of the C3-C5 spinal blocks. Total numbers of CST axons labeled were counted in transverse sections of the $\mathrm{C} 1$ spinal cord. Corticospinal axonal sprouting into the gray matter was quantified in three serial sections containing the dorsal CSTs for each animal. A grid of 0.5 by $0.5 \mathrm{~mm}$ divided into 0.05 by $0.05 \mathrm{~mm}$ boxes was placed over the spinal cord $1 \mathrm{~mm}$ rostral to the lesion. The number of labeled processes crossing each of the 11 vertical lines $0.05 \mathrm{~mm}$ apart, beginning at the gray matter/white matter border, was counted. From this, an overall figure for crossing axons was calculated for each animal by dividing the mean number of CST crossings per section by the number of BDA-traced CST axons. Corticospinal branching within the dorsal white matter was quantified between $\mathrm{C} 3$ and $\mathrm{C} 5$, by dividing the number of branches coming off axons by the number of CST fibers traced. Corticospinal sprouting between the lateral funiculi and gray matter was also quantified by dividing the number of axons crossing the boundary by the number of CST fibers traced (Girgis et al., 2007;
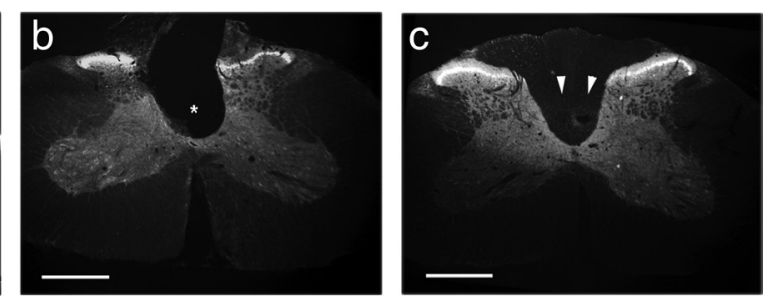

Figure 1. Assessment of the spinal cord lesion. $\boldsymbol{a}$, Transverse section of cervical spinal cord rostral to the dorsal funiculi cord caudal to the spinal cord injury immunostained for $\operatorname{PKC} \gamma$, showing the absence of dorsal corticospinal tract caudal to

García-Alías et al., 2009). Corticospinal axon retraction was quantified by counting the number of the fibers 1.5 and $0.5 \mathrm{~mm}$ rostral to the lesion. This was again normalized against number of traced CST axons at C1.

BDA, vGlut 1 colocalization was quantified in three serial sections containing the dorsal CSTs for each animal. The total number of puncta colabeled with BDA and vGlut $1-1.5 \mathrm{~mm}$ rostral to the lesion was quantified and averaged over the three sections. The data were normalized to the number of BDA axons traced in each animal.

For 5-HT ${ }^{+v e}$ axon, the number of $5-\mathrm{HT}^{+\mathrm{ve}}$ axons crossing a line 0.5 $\mathrm{mm}$ rostral to the lesion was counted in three horizontal sections from layer VII in ventral cord for each animal.

For CGRP ${ }^{+ \text {ve }}$ axon, the number of CGRP ${ }^{+ \text {ve }}$ fibers just rostral to the lesion, crossing the lesion and just caudal to the lesion was quantified in sections where lesions could be easily identified.

\section{Quantification of perineuronal net components}

The intensity of Crtl1, WFA, and aggrecan immunostaining was quantified using a Leica microscope from images captured using the same settings in the same session. The relative intensity of a square region $200 \mu \mathrm{m}$ from the lesion edge with an area of $200 \mu \mathrm{m}^{2}$ was quantified as the ratio against a negative control on the same slide. The number of neurons that had Crtl1 ${ }^{+\mathrm{ve}}, \mathrm{WFA}^{+\mathrm{ve}}$, and aggrecan ${ }^{+\mathrm{ve}}$ perineuronal nets (PNNs) were quantified as a percentage of the total $\mathrm{NeuN}^{+\mathrm{ve}}$ neurons within a square region $200 \mu \mathrm{m}$ from the lesion edge with an area of $40,000 \mu \mathrm{m}^{2}$. The thickness of these PNNs was measured for five randomly chosen neurons on each slide from this region.

\section{Statistical analysis}

Data are shown as the mean \pm SEM. The motor behavioral data were analyzed by two-way ANOVA and Bonferroni post hoc analysis. The sensory behavioral data were analyzed by one-way ANOVA with post hoc analysis. Histological data were analyzed by the Student's $t$ test where there were two groups, and by one-way ANOVA with post hoc analysis for multiple groups.

\section{Results}

\section{Assessment of the lesion after SCI}

Fine forceps were used to cut the dorsal funiculi of the $\mathrm{C} 4$ spinal segments in Lister hooded rats down to the level of the central canal, which disrupted the dorsal columns and the dorsal CST axons, and partially compromised the gray matter of the dorsal and ventral horns. Complete damage to the dorsal CST was verified by protein kinase $\mathrm{C} \gamma(\mathrm{PKC} \gamma)$ staining of transverse sections below the injury at T1 (Fig. $1 a-c$ ) and by observing complete transection of BDA-traced axons at the lesion site. The crosssectional area, width, and depth of all the lesions were calculated. After excluding animals whose lesions did not sever the entire dorsal column/CST, the size and morphology of the cystic cavity formed was similar in all the animals and there were no significant differences between the experimental groups. 

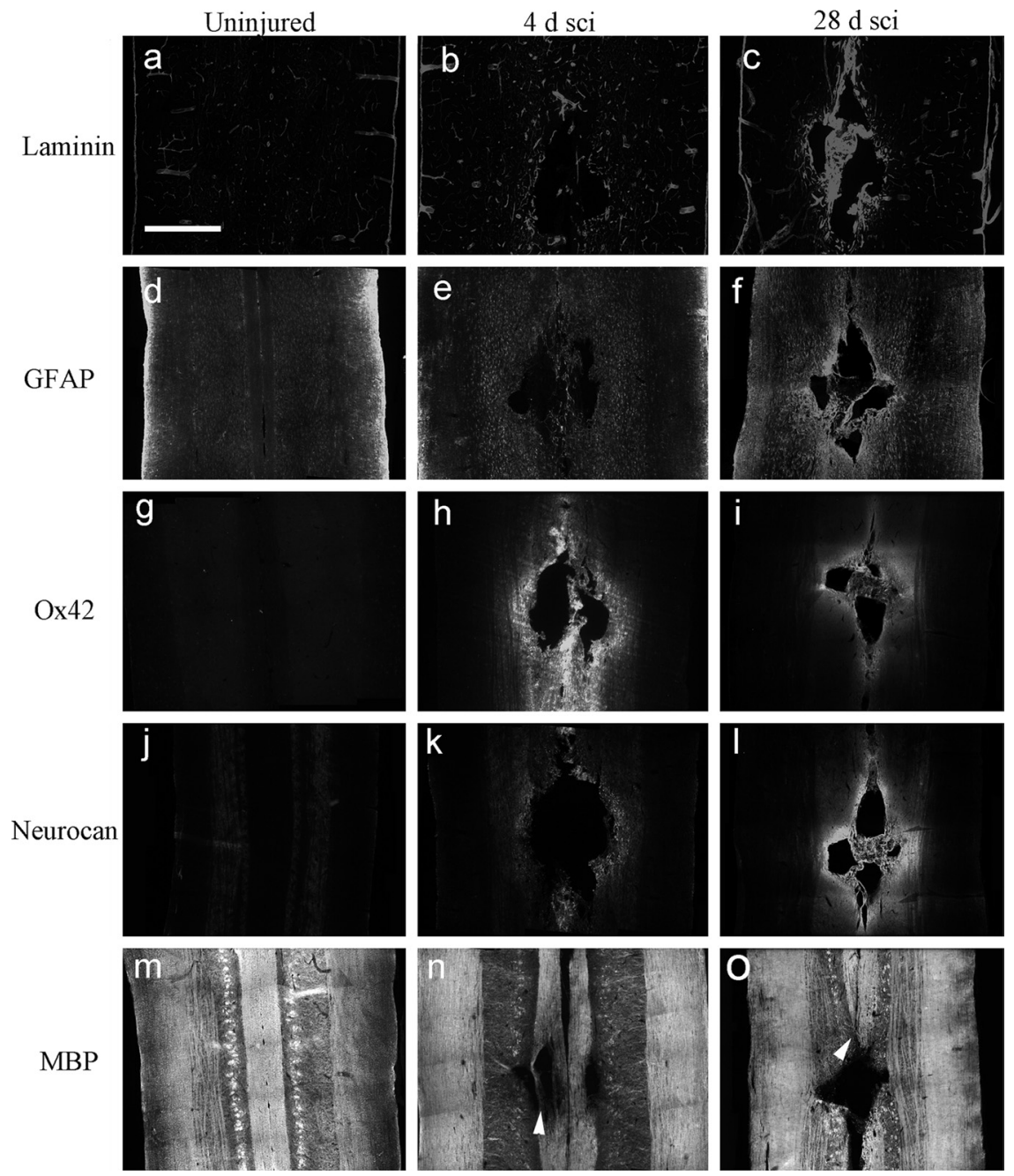

Figure 2. The histological profile of the spinal cord 1 month after dorsal funiculus lesion. $\boldsymbol{a}-\boldsymbol{c}$, Horizontal sections of cervical spinal cord immunostained for laminin with a distinct collagenous scar only present in spinal cord obtained 1 month after injury. $\boldsymbol{d}-\boldsymbol{f}$, Horizontal sections of cervical spinal cord immunostained for GFAP, with a GFAP + ve glial scar more prominent in spinal cord obtained 1 month after injury. $\boldsymbol{g}$-i, Horizontal sections of cervical spinal cord immunostained for 0X42, showing a substantial increase around the lesion area $4 \mathrm{~d}$ after injury, which was much reduced by 1 month. $\mathbf{j}-\mathbf{I}$, Horizontal sections of cervical spinal cord immunostained for neurocan, in spinal cord section obtained $4 \mathrm{~d}$ after injury, upregulation of neurocan was seen perilesionally mostly intracellularly. One month after injury, there was neurocan upregulation within the glial scar both intracellularly and extracellularly. $\boldsymbol{m}-\mathbf{0}$, Horizontal sections of cervical spinal cord immunostained for MBP, with localized myelin loss visible in sections with spinal cord injury (arrowheads). $\boldsymbol{a}, \boldsymbol{d}, \boldsymbol{g}, \boldsymbol{j}, \boldsymbol{m}$, Horizontal sections of normal, intact spinal cord. $\boldsymbol{b}, \boldsymbol{e}, \boldsymbol{h}, \boldsymbol{k}, \boldsymbol{n}$, Horizontal sections of spinal cord $4 \mathrm{~d}$ after injury. $\boldsymbol{c}, \boldsymbol{f}, \boldsymbol{i}, \boldsymbol{I}, \mathbf{0}$, Horizontal sections of spinal cord 1 month after injury. Scale bar, $1 \mathrm{~mm}$.

\section{The 1-month-old lesion had the histological appearance of a chronic injury}

The histological appearance of lesions at $4 \mathrm{~d}(n=3)$ and 1 month ( $28 \mathrm{~d}, n=3$ ) after C4 injury was compared to characterize the chronic lesion environment. Four days after the injury, there was extensive reactivity of microglia and macrophages, as shown by strong OX42 staining around the lesion site, extending $\sim 1-2$ $\mathrm{mm}$ rostral and caudal to the lesion (Fig. $2 g-i$ ). Astrocytes in the same region showed upregulation of GFAP, but had not yet established a border of hypertrophied processes around the lesion (Fig. 2e). Around the lesion cavity, there was increased staining for CSPG neurocan (Fig. $2 j-l$ ). There was also some loss of myelin staining very locally around the lesion site. A mature glial scar with a central core of meningeal-derived cells had not yet been established (Fig. $2 a-c$ ). One month after injury, however, the lesion area appeared to show characteristics of a well established glial scar with a strong perilesional margin of astrocytes expressing high levels of GFAP with hypertrophied and tangled processes around a central fibroblastic core (Fig. $2 a-f$ ). In the same region, there were also high levels of neurocan expression (Fig. $2 j-l$ ). The neurocan staining was both intracellular and extracellular as opposed to a largely intracellular appearance $4 \mathrm{~d}$ after injury. Marked OX42 immunoreactivity remained for $\sim 0.5 \mathrm{~mm}$ around the lesion core, but compared with the acute injury staining, it was less intense and less widespread, suggesting a reduced inflammatory reaction at this time (Fig. 2g-i). Myelin loss was more widespread than in the acute case, with some myelin debris visible in the lesion area (Fig. $2 m-o$ ).

\section{Effective digestion of CSPGs by ChABC in chronic SCI}

Twenty-eight days after the dorsal funiculus injury, animals received two intraparenchymal ChABC $(1 \mu \mathrm{l}, 100 \mathrm{U} / \mathrm{ml})$ or control Pen injections $1 \mathrm{~mm}$ above and below the lesion, followed by bolus intrathecal infusions through a cannula ( $3 \mu \mathrm{l}$, $100 \mathrm{U} / \mathrm{ml}$ ) placed over the lesion every other day for $10 \mathrm{~d}$, as in previous studies. To demonstrate the effectiveness of ChABC digestion, five ChABC and two Pen-treated animals were killed after the last intrathecal infusion. The spinal cord tissues were immunostained for CS-stubs (tetrasaccharide stubs remaining from ChABC digestion) and neurocan. The staining demonstrated that the ChABC had digested the CSPGs throughout the cord 2-3 mm rostral and 3-4 mm caudal to the injury (Fig. 3a). Neurocan had completely disappeared from the digested area, and PNNs were also absent (Fig. $\left.3 b, e^{\prime}, f^{\prime}\right)$. Control Pen injection did not remove the neurocan staining or create stubs (Fig. 3c). There was also digestion in the superficial layer of the dorsal white matter around the cord to a depth of $\sim 100 \mu \mathrm{m}$, extending at least $3 \mathrm{~mm}$ mainly rostral to the lesion (Fig. $3 d^{\prime}$ ).

\section{Animals retained deficits in skilled forelimb functions $28 \mathrm{~d}$ after injury}

The animals' behavior was assessed after injury and before treatment to determine the extent of the deficit, to establish a baseline performance, and to exclude animals showing spontaneous recovery. All animals received training on the staircase task before injury, and only animals that performed successfully on the staircase were included in the study. After injury, these animals received no treatment other than regular observation for $28 \mathrm{~d}$, and were housed with cardboard tubes and wooden blocks as playthings, but no objects that required skilled paw function for their manipulation were present. All animals recovered gross hindlimb and forelimb motor functions within 1 to 2 weeks and, unless challenged with difficult motor tasks, appeared to behave simi- 

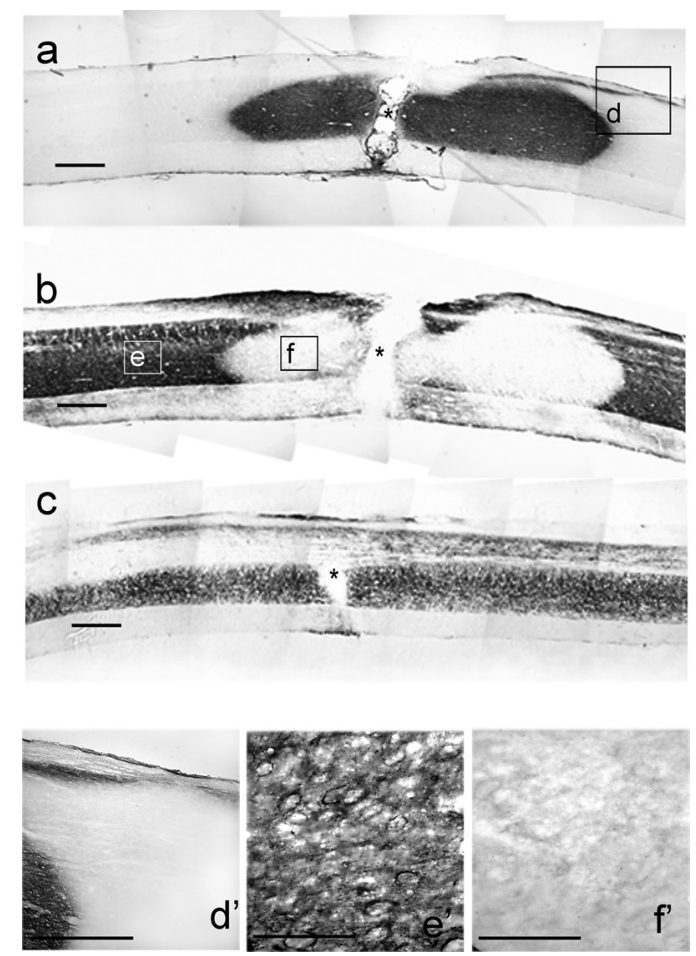

Figure 3. Effective digestion of CSPGs by ChABC in chronic spinal cord injury. $\boldsymbol{a}$, Sagittal section of cervical spinal cord following ChABC treatment 1 month after injury immunostained for $1 \mathrm{~B} 5$ stub; the $1 \mathrm{~B} 5$-positive areas indicating $\mathrm{ChABC}$ diffusion and digestion in both gray and white matter. Scale bar, $1 \mathrm{~mm}$. $\boldsymbol{b}$, Adjacent sagittal section of cervical spinal cord following ChABC treatment 1 month after injury immunostained for neurocan, showing the absence of neurocan 2-3 mm around the injection sites indicating the extent of ChABC diffusion and digestion. Scale bar, $1 \mathrm{~mm}$. c, Sagittal section of cervical spinal cord following penicillinase treatment 1 month after injury immunostained for neurocan, showing neurocan staining throughout the spinal cord, mainly in the gray matter. Scale bar, $1 \mathrm{~mm}$. $\boldsymbol{d}^{\prime}$, Higher magnification of box (d) in $\boldsymbol{a}$, showing the rim of digestion along the dorsal surface of spinal cord. Scale bar, $500 \mu \mathrm{m} . \boldsymbol{e}^{\prime}, \boldsymbol{f}^{\prime}$, Higher magnification of boxes $(\boldsymbol{e}, \boldsymbol{f})$ in $\boldsymbol{b}$ showing the removal of neurocan from the extracellular space and in the perineuronal nets in the region of chondroitinase digestion. Scale bar, $130 \mu \mathrm{m}$.

larly to normal animals. Some forelimb deficits persisted, however, including a considerable deficit in skilled forelimb function. When tested $7 \mathrm{~d}$ after injury, animals had almost completely lost the ability to retrieve sugar pellets on the staircase task (on average, reaching less than one pellet) (Fig. 4a). Most of the animals did not recover significant paw reaching ability and continued to perform very poorly at this task over the next month. There were also other sustained forelimb deficits: animals made more forelimb foot faults on a horizontal ladder and narrow beam, and showed a reduction in forelimb/hindlimb stride length ratio (Fig. $5 b, e, g)$. Four weeks after injury, animals were retested on the staircase paw reaching task, and $\sim 30 \%$ of the animals had regained a significant ability to perform this task, eating four sugar pellets or more. These animals all turned out to have smaller lesions than our standard and were excluded from further experiments. The remaining animals $(n=44)$ were divided into four groups, among which there were no significant behavioral differences (Fig. 4a).

\section{ChABC induced functional recovery in chronic SCI when paired with task-specific rehabilitation}

Animals were divided into four groups, two groups receiving ChABC and two groups Pen; one of the ChABC groups and one of the Pen groups received task-specific rehabilitation, which was begun immediately after treatment in relevant groups (Fig. 4e). The rehabilitation consisted of two $30 \mathrm{~min}$ sessions a day on the skilled paw reaching apparatus retrieving seeds, which were regularly replenished. To assess the effect of treatments on skilled paw reaching, animals were tested weekly on the staircase task (Fig. 4d). Animals receiving ChABC with task-specific rehabilitation began a gradual recovery after treatment, which started to diverge from the control animals around 2 weeks after the commencement of the ChABC injections (Fig. 4a). By 6 weeks after treatment [70 d postinjury (dpi)], these animals (pellets eaten, $4.38 \pm 1.31$ ) were performing significantly better than the other three groups receiving Pen alone (pellets eaten, $0.9 \pm 0.55$ ), ChABC alone (pellets eaten, $0.3 \pm 0.21$ ), or Pen and rehabilitation (pellets eaten, $2 \pm 0.82$ ). The recovery continued over the next few weeks until the last time point ( 9 weeks after treatments, $91 \mathrm{dpi}$ ) when the group receiving $\mathrm{ChABC}$ and rehabilitation performed significantly better than all other groups. They achieved six pellets eaten on average $( \pm 1.52)$, compared with the other three groups, which reached $0.5 \pm 0.40$ (Pen alone, $p<0.001$ ), $0.56 \pm 0.29$ (ChABC alone, $p<0.001$ ), and $2.3 \pm 0.58$ (Pen rehab, $p<0.001$ ) pellets eaten (Fig. $4 a$ ). Overall, the group receiving $\mathrm{ChABC}$ and rehabilitation performed significantly better than all other groups (Pen rehab, $p=0.018$; ChABC alone, $p=$ 0.001; Pen alone, $p=0.001$ ). Animals in the $\mathrm{ChABC}$ rehab group also showed greater accuracy on the staircase apparatus compared with other groups (Fig. $4 b$ ) (Pen rehab, $p=0.045$; ChABC alone, $p=0.022$; Pen alone, $p<0.001)$. The group receiving Pen and rehabilitation showed a small and gradual recovery after rehabilitation started. The recovery was much smaller than that observed in the group that received both $\mathrm{ChABC}$ and rehabilitation, reaching the level of 2.3 pellets eaten on average $( \pm 0.58)$ at the last time point. This recovery was statistically significant when compared with the two groups not receiving rehabilitation. The group receiving ChABC showed signs of recovery during the fourth week after treatment (mean number of pellets eaten increased from $0.9 \pm 0.3$ before treatment to $3 \pm 1.154$ weeks after treatment), but this gain was not sustained. Animals receiving Pen alone did not show signs of recovery at any point.

Similar trends were seen when the accuracy of reaching (the percentage of moved pellets that were successfully eaten) was quantified (Fig. 4b). Both groups that received rehabilitation showed improved accuracy of pellet retrieval compared with the other two groups. However, only the animals that received both rehabilitation and ChABC were able to reach down to the fourth level of the staircase. Animals that received rehabilitation alone only reached down to the second level (Fig. $4 c)(p=$ 0.046, two-way ANOVA with repeated measures between ChABC rehab and Pen rehab). This shows that rehabilitation alone enabled animals to reach the higher-level sugar pellets more accurately, but the combination of $\mathrm{ChABC}$ and rehabilitation enabled animals to reach further as well as more accurately. There was no significant difference in participation in the task between the four groups of animals as the animals did not spend significantly different amounts of time on the platform of the staircase. When animals' skilled forelimb reaching behavior was examined more closely by placing animals into a clear Perspex chamber and filming their grasping of the sugar pellets through an open slit, the animals that received ChABC and rehabilitation appeared to be clumsy; however, they did use the same technique as uninjured animals, reaching with forelimb pronated. 
a

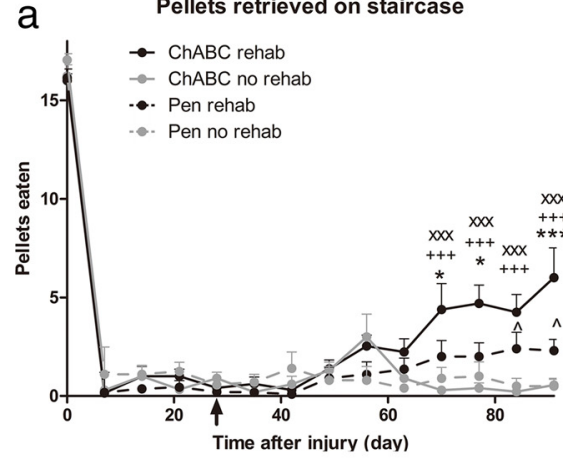

C

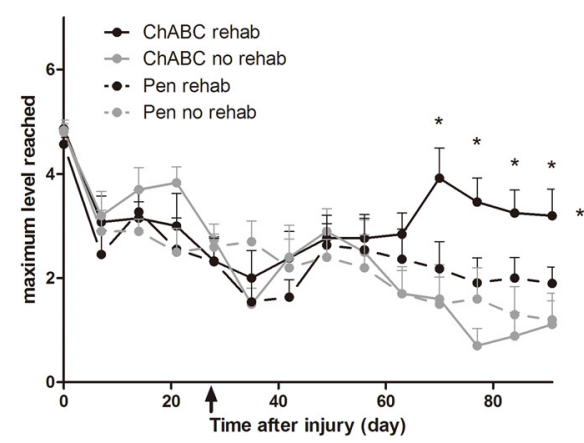

e

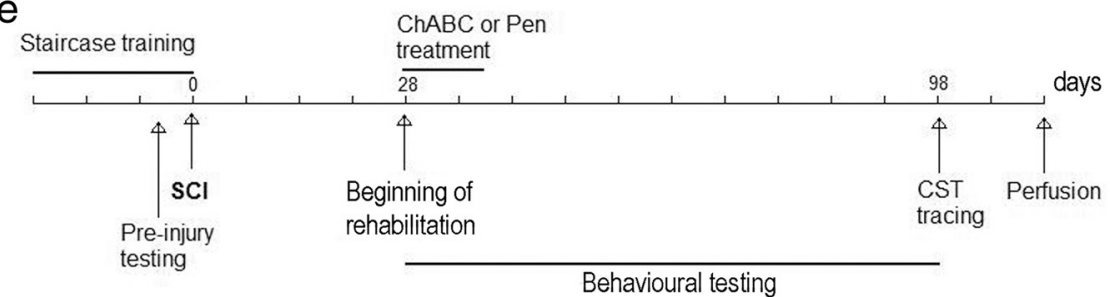

Figure 4. ChABC and task-specific rehabilitation improved the skilled paw reaching of animals with chronic spinal cord injury. $\boldsymbol{a}$, Quantification of the number of pellets eaten on the staircase. C 4 dorsal funiculus injuries were performed at time 0 and $\mathrm{ChABC}$ or Pen was injected at $28 \mathrm{~d}$ (arrowhead) when rehabilitation started. There was little recovery in any of the groups during the month after injury (days $7,14,21$, and 28). After the treatment, the group receiving $\mathrm{ChABC}$ and rehabilitation performed significantly better than all other groups at later time points (days 70,77, 84, and 91). ${ }^{*} p<0.05 \mathrm{ChABC}$ rehab versus Pen rehab; ${ }^{* * *} p<$ $0.001 \mathrm{ChABC}$ rehab versus Pen rehab; ${ }^{\mathrm{xxx}} p<0.001$ ChABC rehab versus ChABC no rehab; ${ }^{++} p<0.001 \mathrm{ChABC}$ rehab versus Pen no rehab. Overall, the group receiving $C \mathrm{CABC}$ and rehabilitation performed significantly better than all other groups (Pen rehab, $p=0.018$; (hABC alone, $p=0.001$; Pen alone, $p=0.001$ ). The group receiving Pen and rehab also showed a gradual recovery, although not to the level of $\mathrm{Ch} A \mathrm{BC}$ with rehab; this group performed significantly better at the last two time points when compared with the pooled no-rehab groups. ${ }^{x} p<0.05$, Pen rehab versus $C h A B C$ no rehab and Pen no rehab. $\boldsymbol{b}$, Similar trends were observed for accuracy (the number of pellets eaten/total number displaced) of performance. The animals receiving $C h A B C$ and rehab were significantly more accurate at grasping sugar pellets than other groups. ${ }^{x} p<0.05$ ChABC rehab versus $C h A B C$ no rehab; ${ }^{x x} p<$ $0.01 \mathrm{ChABC}$ rehab versus ChABC no rehab; ${ }^{\mathrm{xxx}} p<0.001 \mathrm{ChABC}$ rehab versus $\mathrm{ChABC}$ no rehab; ${ }^{++} p<0.01 \mathrm{ChABC}$ rehab versus Pen no rehab; ${ }^{+++} p<0.001 \mathrm{ChABC}$ rehab versus Pen no rehab. Overall, the group receiving $\mathrm{ChABC}$ and rehabilitation was significantly more accurate than all three other groups (Pen rehab, $p=0.045$; ChABC alone, $p=0.022$; Pen alone, $p<0.001$ ). Animals treated with ChABC also showed improvement in accuracy of grasping up to day 56 ( ${ }^{\# \#} p<0.01$ ChABC no rehab vs Pen no rehab), which was not sustained. This trend was also observed with number of pellets eaten; however, it was not significant at this time point. All statistics unless otherwise stated were performed with two-way ANOVA with repeated measures and post hoc analysis. c, Animals receiving $\mathrm{ChABC}$ and rehab also reached further than animals in other groups. Maximum distance reached by animals receiving $\mathrm{ChABC}$ and rehab is significantly higher than the animals receiving Pen and rehab $\left({ }^{*} p<0.05\right.$, two-way ANOVA with repeated measures between $\mathrm{Ch} A B C$ rehab and Pen rehab) over the last six time points; as well as over individual time points (days $70,77,84$, and 91 ). ${ }^{*} p<0.05$, Student's $t$ test. $\boldsymbol{d}$, The staircase apparatus. Animals slide into the tunnel, gaining access to the left and right staircases. They grasp sugar pellets for $15 \mathrm{~min}$, and the numbers of sugar pellets displaced and eaten are quantified separately. $\boldsymbol{e}$, A timeline of the experiment.

\section{$\mathrm{ChABC}$ and rehabilitation promote recovery in other related forelimb functions}

Forelimb functions other than skilled paw reaching were also affected by $\mathrm{C} 4$ dorsal funiculi lesions. The effect of $\mathrm{ChABC}$ and rehabilitation on other forelimb functions was investigated. An- imals made more forelimb slips when walking along horizontal ladders and beams after injury, and these deficits had not recovered at the onset of treatment 4 weeks after injury (Fig. 5b,e). At the end of rehabilitation, the group receiving $\mathrm{ChABC}$ and rehabilitation made significantly fewer mistakes on the horizontal ladder when compared with groups without rehabilitation $(15.4 \pm 1.04$ vs $20.8 \pm 0.93$, ChABC alone, $p=0.006$; or $20.7 \pm 0.97$, Pen alone, $p=0.005$ ) (Fig. $5 b$ ). Animals treated with $\mathrm{ChABC}$ and rehabilitation also showed a reduction in the percentage of mistakes (Fig. 5c). Animals receiving Pen and rehabilitation also made significantly fewer mistakes, recovering to the same extent as animals treated with $\mathrm{ChABC}$ and rehabilitation $(16.2 \pm 1.03 ; p=0.029$, Pen rehab vs ChABC alone; or $p=0.025$, Pen rehab vs Pen alone), although the percentage of mistakes was not significantly improved (Fig. 5c). Rehabilitation similarly improved the accuracy of the animals' forelimb placement while walking along a horizontal beam. Here again, both the ChABC rehab and Pen rehab groups performed better than the animals that did not receive rehabilitation (Fig. $5 d, e$ ).

In previous experiments using this lesion, we saw a change in gait, with the animals making small frequent forelimb steps while maintaining longer hindlimb steps, and animals treated with ChABC recovered a more normal stride length than those receiving control penicillinase (García-Alías et al., 2008). In the current experiment, the forelimb/hindlimb stride length ratio decreased after injury as before, due to a decrease in forelimb stride length and compromised forelimb, hindlimb coordination. However, neither ChABC nor rehabilitation changed the forelimb/hindlimb stride length ratio (Fig. $5 f, g$ ) or the base of support of these animals' gait. We also measured grip strength using a device that measures the traction force exerted on hand bars before animals lose their grip. There was no difference in forelimb grip strength between any treatment groups, all groups having recovered to normal levels 4 weeks after injury. These results, together and compared with our previous results using the same lesion, show that in chronic injured spinal cord, complex tasks such as skilled paw reaching, which are heavily dependent on the CST, only recover with $\mathrm{ChABC}$-induced plasticity combined with rehabilitation. Other less skilled tasks such as ladder walking, which involve other spinal pathways such as reticulospinal tracts (spared in this model) as well, can recover with rehabilitation alone. Other behaviors such as grip strength and gait are not changed by forelimb reaching rehabilitation. 
a

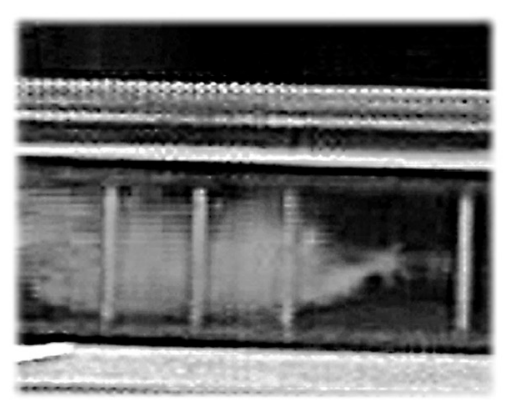

b

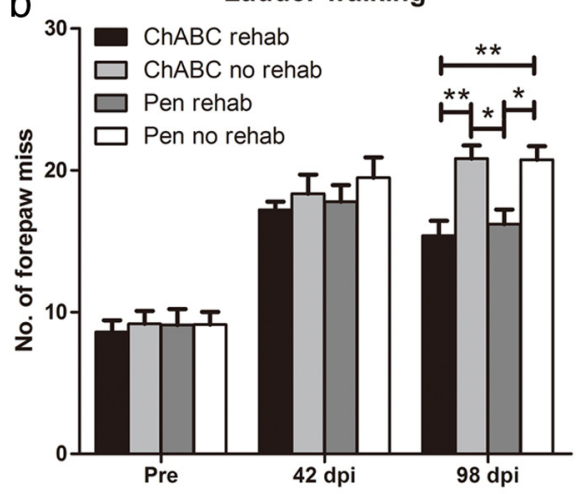

C

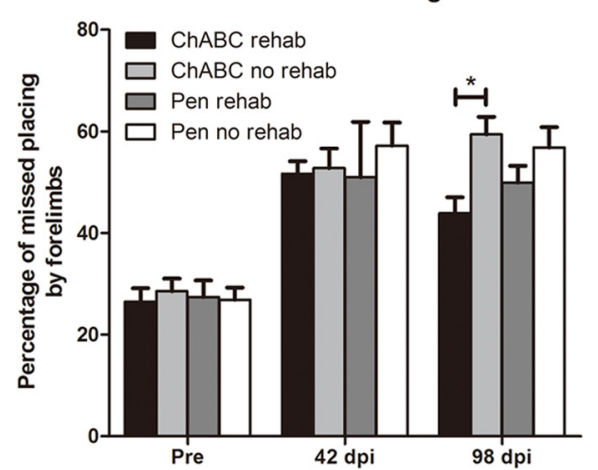

d

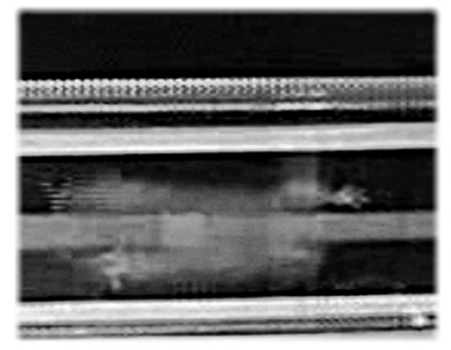

e

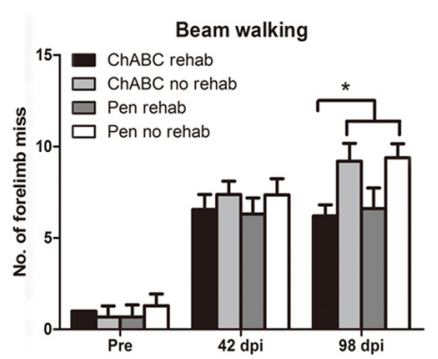

$f$

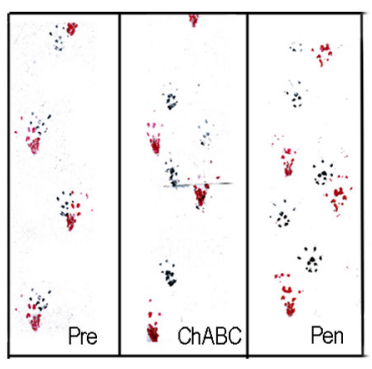

g

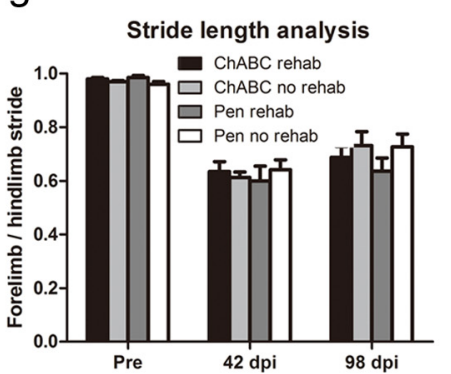

Figure 5. Both ChABC and task-specific rehabilitation and task rehabilitation alone improved tasks closely related to skilled paw reaching in animals with chronic spinal cord injury. $\boldsymbol{a}$, Horizontal ladder; an animal was unable to place its right forepaw onto the ladder correctly while walking across it. $\boldsymbol{b}$, After injury, the animals made significantly more forepaw slips while walking on the horizontal ladder, which was sustained 1 month after the injury (day 42, $p<0.001$ ). By day 98, the animals receiving ChABC and rehab made significantly fewer mistakes compared with animals receiving $C h A B C$ or Pen without rehab. ${ }^{* *} p<0.01$, ChABC rehab was compared with $C h A B C$ no rehab or Pen no rehab. The animals receiving rehabilitation alone also made significantly fewer mistakes compared with animals receiving $\mathrm{ChABC}$ or Pen without rehab. ${ }^{*} p<0.05$, Pen rehab was compared with ChABC no rehab or Pen no rehab. $c$, Quantification of percentages of slips on horizontal ladder. After injury, animals made proportionally more forepaw slips while walking on the horizontal ladder, which was sustained 1 month after injury (day $42, p<0.001$ ). By day 98 , the animals receiving $\mathrm{ChABC}$ and rehab made significantly fewer mistakes compared with animals receiving $\mathrm{ChABC}$ without rehab. ${ }^{*} p<0.05$. $\boldsymbol{d}$, Horizontal beam, an animal was unable to place its paws onto the narrowing beam. This test again evaluates the abilities of animals to place their forepaws correctly. $\boldsymbol{e}$, After injury, the animals made significantly more forepaw slips while walking on the horizontal beam, which was sustained 1 month after the injury (day 42, $p<0.001$ ). Rehabilitation seemed to have the most effects on animals' ability to walk across the beam, with animals receiving $C h A B C$ and rehab making significantly less mistakes by day 98 when compared with ChABC no rehab and Pen no rehab combined $\left({ }^{*} p<0.05\right)$. $f$, Typical footprints of animals walking. Forelimb/hindlimb stride length ratio was used to assess the coordination of gait. Before injury (Pre), animals' forelimb and hindlimb stride length was similar. After injury, regardless of the treatment, animals made more forelimb steps than hindlimb steps (ChABC, Pen). Black: forepaw footprints; Red: hindpaw footprints. $\boldsymbol{g}$, After injury, there was a sustained reduction in forelimb/ hindlimb stride length ratio (day $42, p<0.001$ ). None of the treatments significantly changed the ratio between days 42 and 98 . All statistics unless otherwise stated were performed with two-way ANOVA with repeated measures and post hoc analysis.

\section{ChABC treatment and specific rehabilitation did not cause abnormal pain sensation}

It has been suggested that increased plasticity might increase the level of aberrant sprouting leading to abnormal sensation and hyperalgesia; however, neither ChABC nor task-specific rehabilitation affected the threshold for pain, temperature, and pressure sensation. After injury, the animals showed reduced pain and temperature sensation. These deficits persisted 1 month after injury and were still present at the end of rehabilitation and behavioral studies. On average, they took $6.86 \pm 0.33 \mathrm{~s}$ to respond to a heat source compared with $4.63 \pm 0.29 \mathrm{~s}$ with sham animals (Fig. $6 a)$. They also had a deficit in their response to pressure sensation, responding to $80.6 \pm 2.76 \times g$ of pressure compared with $52.1 \pm 2.83 \times g$ with sham animals (Fig. 6b). There was no significant difference between all treatment groups indicating that neither ChABC nor rehabilitation caused allodynia or hyperalgesia.

\section{$\mathrm{Ch} \mathrm{ABC}$ promotes sprouting and new connections in} chronically injured spinal cord

Corticospinal axons (CST)

The anatomical changes in the injured dorsal CST were studied following bilateral BDA injection into the forepaw representation area of the sensorimotor cortex. In all animals, the dorsal CST had been completely cut at the lesion site (Fig. $7 a, c$ ). We quantified dorsal CST sprouting by counting the number of sidebranches made by CST axons in the dorsal white matter and by counting the number of labeled CST processes crossing the gray/ white matter boundary $1-1.5 \mathrm{~mm}$ rostral to the lesion (Fig. $\left.7 b^{\prime}, d^{\prime}\right)$. Both groups receiving $\mathrm{ChABC}$ regardless of rehabilitation showed increased axon branching and crossing compared with control Pen-treated groups (Fig. 7e,f). In both the Pen and ChABC groups, the means for branching and crossing of CST axons were higher in the animals that received rehabilitation, but this trend was not statistically significant. Branching from the unlesioned dorsolateral CST was also quantified as an increase had previously been observed following ChABC treatment after acute SCI. The results were very variable, and there were no significant treatment effects on this measure.

On initial observation, the BDA-labeled axons appeared to be closer to the lesion site in ChABC-treated animals (Fig. $7 h^{\prime}-m^{\prime}$ ). We therefore quantified the number of axons within $0.5 \mathrm{~mm}$ to the lesion edge and found that there were significantly more axon fibers near to the lesion in the group receiving $\mathrm{ChABC}$ and rehabilitation than in other groups (Fig. $7 g$ ). Further rostrally, at 1.5 
a

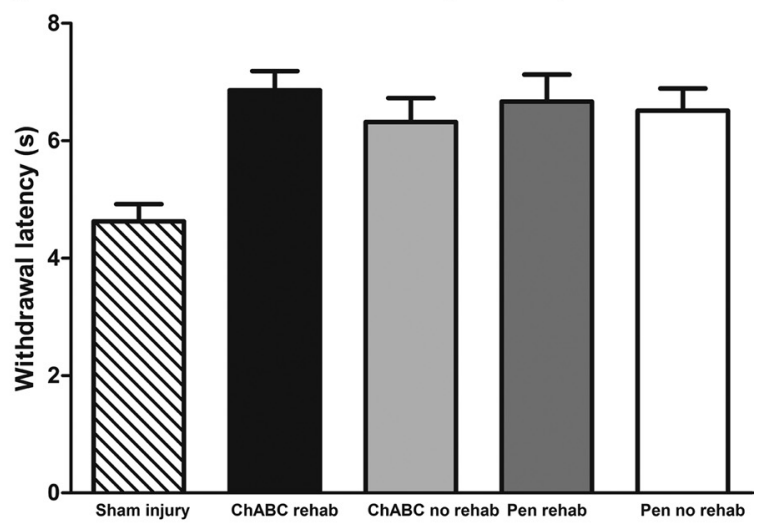

b

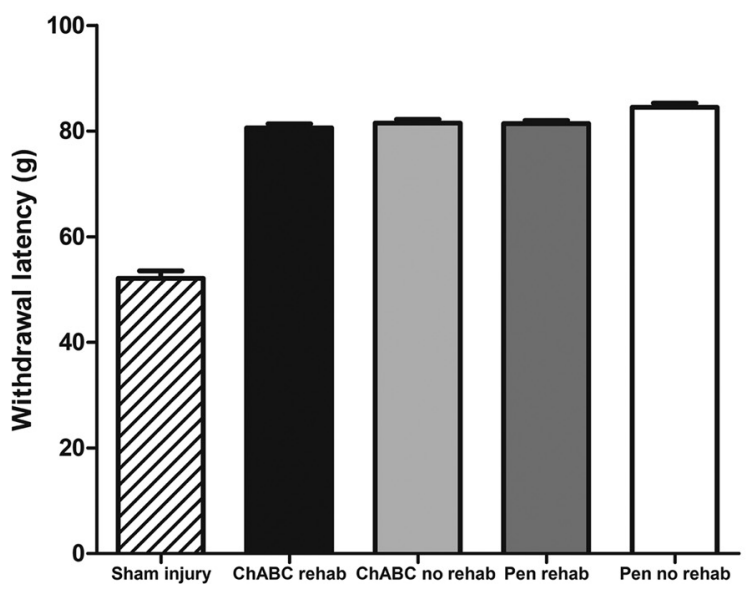

Figure 6. Neither $\mathrm{ChABC}$ nor task-specific rehabilitation caused abnormal pain sensation. $\boldsymbol{a}$, Hotplate analysis was used to test the animals' ability to respond to thermal stimuli. After injury, the animals took significantly longer to respond to an infrared heat source when compared with sham-injured animals ( $p<0.01$, sham vs all-treatment groups). There was no significant difference between the treatment groups, indicating that $\mathrm{ChABC}$ or task-specific rehabilitation did not change or cause abnormal sensation in response to a thermal stimulus. $\boldsymbol{b}$, An electronic anesthesiometer was used to test the animals' abilities to respond to pressure stimuli. After injury, higher pressure was needed to induce withdrawal of the animals' forepaws when compared with sham-injured animals ( $p<0.001$, sham vs all-treatment groups). There was no significant difference between the treatment groups, indicating that $\mathrm{ChABC}$ or task-specific rehabilitation did not cause abnormal sensation in response to a pressure stimulus.

$\mathrm{mm}$ from the lesion there were no differences in axon number between the groups, indicating that the larger number of axons rostral to the lesion in the $\mathrm{ChABC}$ rehabilitation group was very local to the lesion. Because ChABC was applied starting 1 month after injury, it is unlikely that the larger number of axons near the lesion was due to axonal protection. It is more probable that these are processes that had sprouted from axons that retracted away from the lesion at the time of injury. We did not see any axons that had grown around or past the lesion site, or through the scar tissue surrounding the injury.

$B D A^{+v e}$ and $v G l u t 1^{+v e}$ synaptic puncta

Sprouting from the CST axons could not have affected behavior unless synapses were formed. To see whether the axon sprouts induced by $\mathrm{Ch} A B C$ treatment make connections, we assessed the number of $\mathrm{vGlut}^{+\mathrm{ve}}$ puncta on the $\mathrm{BDA}^{+\mathrm{ve}}$ corticospinal sprouts in the gray matter. A synaptic varicosity was usually present where a patch of vGlut 1 staining coincided with a BDA ${ }^{+v e}$ process (Fig. $8 a-c$ ). Both groups receiving $\mathrm{ChABC}$ had signifi- cantly more $\mathrm{BDA}^{+\mathrm{ve}}$, vGlut ${ }^{+\mathrm{ve}}$ synaptic puncta than the groups receiving Pen (Fig. 8d). Both groups that received rehabilitation appeared to have more colocalized puncta than in the respective groups that had not received rehabilitation. This was most noticeable in the case of ChABC-treated animals, where the group with rehabilitation appeared to have many more colocalized puncta. As with the similar trend in CST sprouting, this trend was not statistically significant here. However, it could potentially indicate that rehabilitation encouraged establishment of functional synapses on axonal sprouts.

\section{Serotonergic and sensory axons}

The sprouting of other CNS axons was also assessed to study whether or not sprouting was restricted to CSTs. Serotonergic axons were visualized by anti-5-HT antibody staining. There was an increase in the number of $5-\mathrm{HT}^{+\mathrm{ve}}$ axons around the lesion site after injury in all the experimental groups compared with regions of the cord further away. The number of axons crossing a line $0.5 \mathrm{~mm}$ rostral to the lesion was quantified, showing that animals that received ChABC injections (with or without rehabilitation) had significantly more axons rostral to the lesion, indicating an increase in the sprouting of serotonergic axons after ChABC treatment $(p=0.022)$ (Fig. 8e). CGRP ${ }^{+v e}$ sensory axons were also visualized by anti-CGRP immunostaining, and the number of axons rostral to the lesion, in the lesion epicenter, and caudal to the lesion were quantified. No significant differences were found between the groups (data not shown).

\section{Task-specific rehabilitation increased the extracellular components of PNNs}

To address the mechanism behind the interaction of rehabilitation and the ECM, we investigated the effect of the interventions in our experiment and of delayed task-specific training on PNNs. ChABC treatment has been shown previously to remove all WFA staining, and to partially remove link protein and aggrecan staining, which was indeed the case here for spinal cords at the end of the ChABC treatment period (Fig. 9j-m). We examined the spinal cords from animals after the end of the study, 18 weeks after SCI, 12 weeks after the last ChABC injection and 2 weeks after the final rehabilitation session. We saw no significant differences in the number of PNNs or their intensity of staining for WFA, Crtll link protein, or aggrecan (data not shown). This indicates that the PNN molecules removed by ChABC treatment had been replaced during this time, and that any differences caused by rehabilitation had normalized. To assay the effect of rehabilitation in PNNs, we therefore examined animals that had received a $\mathrm{C} 4$ dorsal column lesion then continuous rehabilitation or no rehabilitation for 18 weeks. The cervical spinal cord sections caudal to the lesion of these animals were immunostained for extracellular components of the following PNNs: Crtl1, WFA, aggrecan, and hyaluronan synthase-3 (Fig. 9). We observed a rehabilitation-dependent increase in Crtll and WFA levels in these animals (Fig. $9 a-i, n$ ) as well as an increase in the number and thickness of Crtll-positive PNNs throughout the cervical spinal cord (Fig. 9o,p). One of the authors (R.M.I.) has observed a similar result in animals that received lower limb rehabilitation after complete cord transection (unpublished data).

\section{Discussion}

Any intervention for SCI must work alongside rehabilitation and other routine treatments. Our previous study showed that ChABC treatment greatly enhances the effect of rehabilitation. We now show that combined ChABC and rehabilitation is effi- 

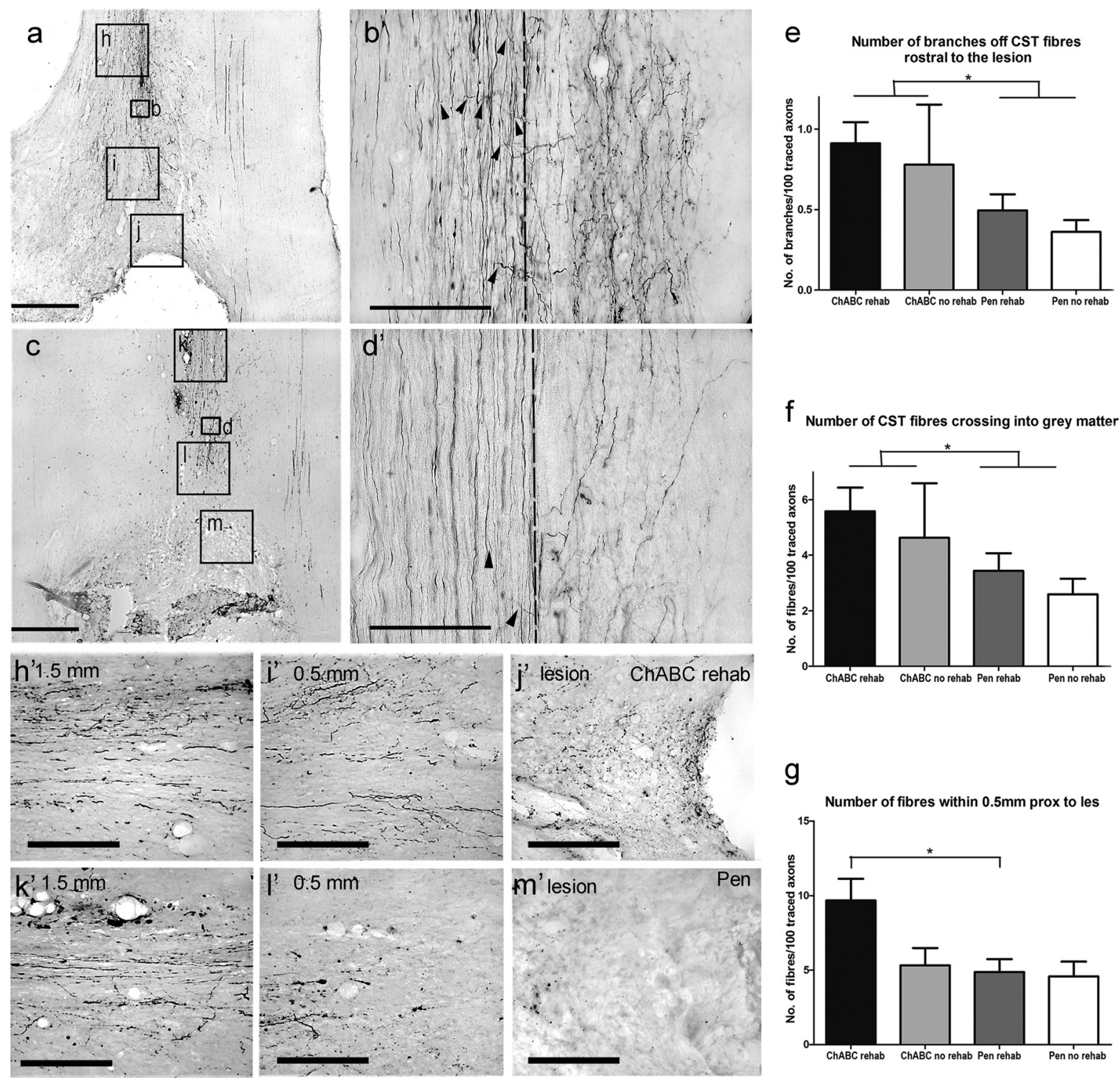

Figure 7. ChABC promotes sprouting in chronically injured spinal cord. $\boldsymbol{a}$, Horizontal section of cervical spinal cord from an animal that received ChABC and task-specific rehabilitation 1 month after injury; the CST was traced with BDA and stained using the DAB method. There was extensive sprouting of the severed CST rostral to the lesion into the gray matter. Scale bar, $500 \mu \mathrm{m} . \boldsymbol{b}^{\prime}$, Higher magnification of box (b) in $\boldsymbol{a}$, showing the extensive sprouting (-, the gray/white matter boundary; $\uparrow$, branching off dorsal (ST in white matter). Scale bar, $100 \mu \mathrm{m}$. $\boldsymbol{c}$, Horizontal section of cervical spinal cord from an animal that received Pen and task-specific rehabilitation 1 month after injury. There was much less sprouting of the severed CST rostral to the lesion into the gray matter. Scale bar, $500 \mu \mathrm{m} . \boldsymbol{d}^{\prime}$, Higher magnification of box $(\boldsymbol{d})$ in $\boldsymbol{c}$, showing less sprouting (-, the gray/white matter boundary; $\uparrow$, branching off dorsal CST in white matter). Scale bar, $100 \mu \mathrm{m} . \boldsymbol{e}, \boldsymbol{f}$, Quantification of CST axonal sprouting rostral to the lesion both by quantifying number of branches in the white matter $(\boldsymbol{e})$ and sprouting into the gray matter $(\boldsymbol{f})$ in all four treatment groups. ChABC injection significantly increased the number of axon branches and sprouts. ${ }^{*} p<0.05$. The apparent trend of increased sprouting in the rehab groups was not statistically significant. $\boldsymbol{g}$, Quantification of CST axons within $0.5 \mathrm{~mm}$ of the lesion in all four treatment groups. ChABC and rehabilitation combined treatment lead to more fibers closer to the lesion. ${ }^{*} p<0.05 . \boldsymbol{h}^{\prime}-\boldsymbol{j}^{\prime}$, Higher-magnification pictures of the boxes $(\boldsymbol{h}-\boldsymbol{j})$ in horizontal section of an animal received ChABC and rehabilitation $(\boldsymbol{a})$ showing the lesion site $\left(\boldsymbol{j}^{\prime}\right), 0.5 \mathrm{~mm}$ rostral to lesion $\left(\boldsymbol{i}^{\prime}\right)$ and $1.5 \mathrm{~mm}$ rostral to lesion $\left(\boldsymbol{h}^{\prime}\right)$. BDA ${ }^{+ \text {ve }}$ axons were seen extensively at all three positions. Scale bar, $200 \mu \mathrm{m} . \boldsymbol{k}^{\prime}-\boldsymbol{m}^{\prime}$, Higher-magnification pictures of the boxes $(\boldsymbol{k}-\boldsymbol{m})$ in horizontal section of an animal treated with Pen and task-specific rehabilitation $(\boldsymbol{c})$ showing the lesion site $\left(\boldsymbol{m}^{\prime}\right), 0.5 \mathrm{~mm}$ rostral to lesion $\left(\boldsymbol{I}^{\prime}\right)$, and $1.5 \mathrm{~mm}$ rostral to lesion $\left(\boldsymbol{k}^{\prime}\right)$. BDA ${ }^{+ \text {ve }}$ axons were seen extensively at $1.5 \mathrm{~mm}$ rostral to lesion $\left(\boldsymbol{k}^{\prime}\right)$; however, fewer were seen at $0.5 \mathrm{~mm}$ rostral to the lesion $\left(\boldsymbol{I}^{\prime}\right)$ and at the lesion site $\left(\boldsymbol{m}^{\prime}\right)$. Scale bar, $200 \mu \mathrm{m}$.

cacious in chronic SCI, and might therefore be started when patients usually begin rehabilitation. Many additional barriers to the repair of chronic and subacute SCIs do not exist for acute treatments. There is neuronal cell death and atrophy as well as extensive axonal degeneration and demyelination. The optimum window for axonal regeneration may also have passed (Schwab and Bartholdi, 1996), and the glial scar around the injury becomes established and dense (Rudge and Silver, 1990; Stichel and Muller, 1994; Silver and Miller, 2004). Many regenerative interventions are effective in acute SCI but not in a chronic setting
(Shumsky et al., 2003; López-Vales et al., 2006, 2007; Nishio et al., 2006).

We have used a cervical SCI model that ablates both dorsal CSTs and parts of the dorsal column. The motor cortex and CSTs are critical for motor control in humans; we therefore used an animal model in which recovery of CST function is the main outcome measure (Bradbury et al., 2002; García-Alías et al., 2008, 2009). Ablation of the CST affects manual dexterity in many animals, including rats, leading to behavioral deficits in skilled paw manipulation that can be assessed by reaching tasks 

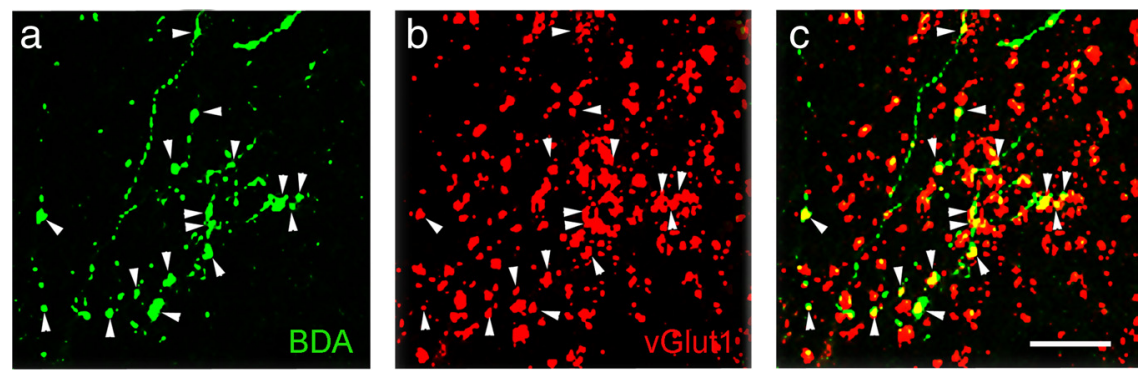

d

vGlut1 ${ }^{\text {vee }}, \mathrm{BDA}^{\text {+ve }}$ punta

e
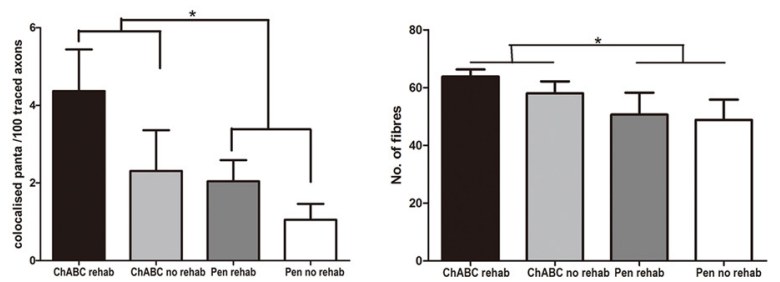

Figure 8. vGlut1 colocalizes with the corticospinal axon sprouting. $\boldsymbol{a}-\boldsymbol{c}$, Fluorescence images showing the vGlut1 ${ }^{+v e},{ }_{B} B A^{+v}$ synaptic puncta. Scale bar, $20 \mu \mathrm{m}$ ( $\uparrow$, vGlut $1^{+v e}$, BDA $^{+ \text {ve }}$ synaptic puncta). $\boldsymbol{d}$, Quantification of vGlut1 ${ }^{+v e}$, BDA $^{+ \text {ve }}$ synaptic puncta rostral to the lesion in the four treatment groups. ChABC injection significantly increased the number of colocalized puncta. ${ }^{*} p<0.05$. There is an apparent trend of increased number of colocalized puncta with training, although this is not statistically significant. e, Quantification of 5-HT axons in layer VII of the spinal cord gray matter rostral to the lesion. ChABC injection significantly increased the number of $5-\mathrm{HT}{ }^{+ \text {ve }}$ axons. ${ }^{*} p<0.05$.

(Whishaw et al., 1993, 1998; Basso et al., 1996; McKenna and Whishaw, 1999; Starkey et al., 2005; Pettersson et al., 2007). After injury, animals recover most gross motor functions rapidly, but show a sustained deficit in skilled paw reaching (Bradbury et al., 2002; García-Alías et al., 2008, 2009).

Aiming to design a regimen suitable for future treatment of human patients, we chose the time point of 1 month after injury to begin treatment. One month is an appropriate time point in human SCI management as patients are stabilized and rehabilitation has usually begun. At this time point, injury sites had the appearance of a chronic lesion. There was a well established glial scar with CSPG upregulation, and the acute inflammatory responses had ended. Others have observed similar changes (Stichel and Muller, 1994; Hu et al., 2010), although evidence exists that pathology continues to develop until 14 weeks after injury in rodents (Hill et al., 2001). In our study and others, behavioral recovery plateaus 4 weeks after injury (Basso et al., 1995); therefore, it is commonly used as a reasonable time point to represent chronic SCI (Coumans et al., 2001; Lu et al., 2002; Houle and Tessler, 2003; Nishio et al., 2006).

Several studies have shown that ChABC treatment promotes functional recovery after acute SCI (Bradbury et al., 2002; Caggiano et al., 2005; Tester and Howland, 2008). Greater recovery of CST function occurred when ChABC was paired with specific rehabilitation, as would occur in a clinical setting (García-Alías et al., 2009), the effects of rehabilitation being focused on the precise task that was reinforced. We therefore rehabilitated animals for $1 \mathrm{~h}$ daily through grasping of seeds from the staircase skilled paw reaching apparatus. We used the same task, loaded with sugar pellets, as the main outcome measure. Functional recovery was delayed compared with our previous study, becoming significant after 6 weeks, but visibly deviating from controls 2 weeks after the first treatment. By 9 weeks, animals had recovered CST function almost equivalent to our previous acute treatment experiment. Rehabilitation alone slightly improved the animals' performance on the staircase, both increasing the number of attempts and their accuracy; however, animals still only reached down to the second stair of seven. Animals treated with $\mathrm{ChABC}$ and rehabilitation were able to reach the fourth stair, and to do so accurately. We conclude that ChABC opened a window of plasticity in chronically injured spinal cord during which specific rehabilitation was able to drive substantial recovery in manual dexterity.

There is increasing evidence that different behaviors compete for the available neurological resources after SCI, so that intensive rehabilitation of one task may improve performance of that task at the expense of others. Spinally transected cats can be trained in either weight support or stepping, but successful stepping removes weight support and vice versa (De Leon et al., 1998a,b); rodents trained for skilled reaching make more mis-steps when running on a ladder, and general environmental enrichment extinguishes paw reaching (Girgis et al., 2007; García-Alías et al., 2009; Krajacic et al., 2010). After a unilateral cortical lesion, training the intact paw compromises recovery of the injured paw (Allred and Jones, 2008). In the present study, however, rehabilitation of skilled paw function did not produce deficits in other motor tasks tested. Furthermore, the groups of animals receiving rehabilitation performed better in some tasks, making fewer mistakes while running along a ladder and beam. Similar results have been shown previously; after unilateral CST lesions, rats receiving delayed skilled grasping training also improve their performance in ladder walking (Krajacic et al., 2010). We did not repeat our previous experiment in which general environmental enrichment competed with skilled paw reaching, and paw-reaching rehabilitation did not affect performance on the ladder task in that study (García-Alías et al., 2009). Together, these results imply that in chronic SCI complex tasks such as skilled paw reaching, which rely heavily on the CST, only recover with ChABC-induced plasticity combined with rehabilitation. Less skilled tasks such as ladder walking, which also involve other spinal pathways such as reticulospinal tracts, recover with rehabilitation alone.

How might ChABC and rehabilitation work together to promote functional recovery? ChABC digestion changes the CNS ECM, leading to three main effects. After acute injury, there is increased axonal regeneration (Bradbury et al., 2002; Yick et al., 2003; Caggiano et al., 2005; Iseda et al., 2008). However, in our chronic model the glial scar was firmly established and ChABC treatment did not produce CST regeneration past the lesion. The beneficial behavioral effects are therefore unlikely to have been caused by long-distance axonal regeneration. The second effect is sprouting of axons above and below the injury (Bradbury et al., 2002; Moon et al., 2002; Barritt et al., 2006; Massey et al., 2006; Cafferty et al., 2008; García-Alías et al., 2009; Tom et al., 2009b). We saw CST sprouting of a similar magnitude to that in acutely treated cord, and also sprouting of serotonergic axons. These axon sprouts made more synapses in ChABC-treated animals. We did not see sprouting of unlesioned CST axons below the lesion, so the new connections and behavior must have been due to CST sprouts above the lesion synapsing onto propriospinal interneurons projecting below the lesion. The third effect of 

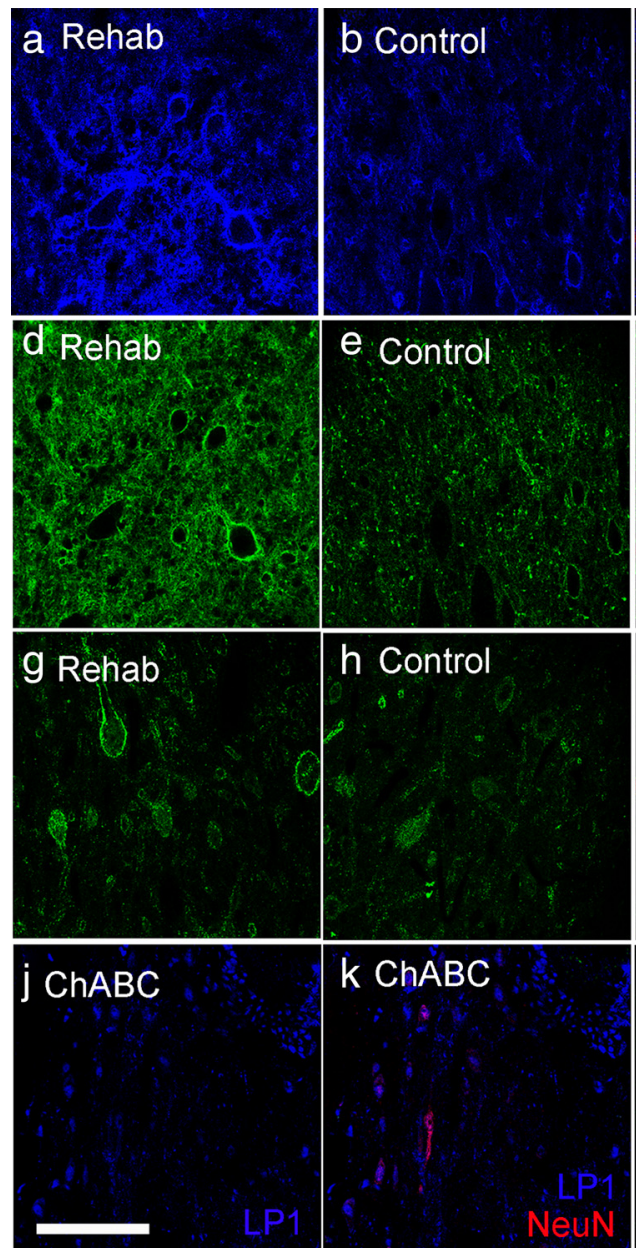
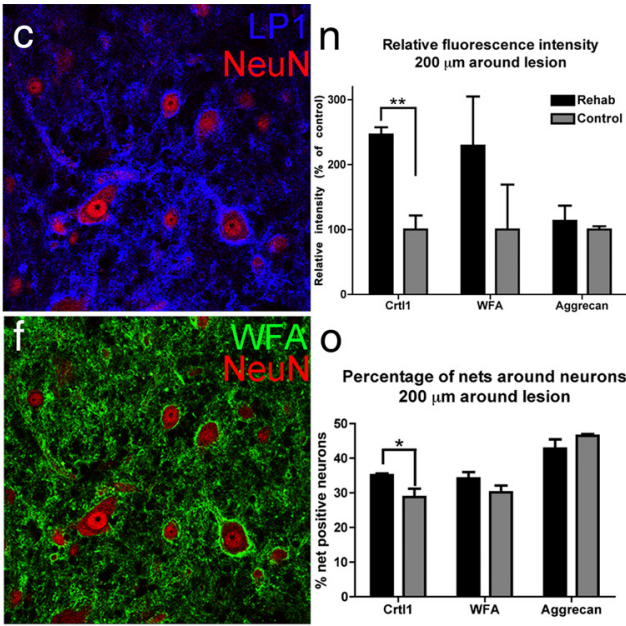
$200 \mu \mathrm{m}$ around lesion
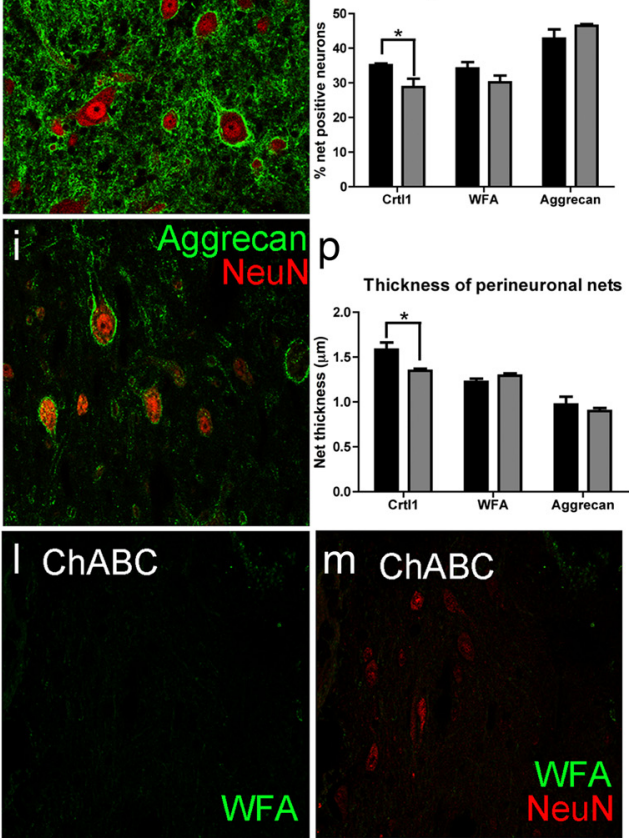

Figure 9. Rehabilitation upregulated components of PNNs after spinal cord injury. $\boldsymbol{a}-\boldsymbol{c}$, Fluorescence images of ( $\mathrm{rt}$ 11 $200 \mu \mathrm{m}$ from the lesion site, with (a) or without any rehabilitation (control, $\boldsymbol{b})$ and colocalization with neurons showing PNN structures (c). $\boldsymbol{d}-\boldsymbol{f}$, Fluorescence images of WFA $200 \mu \mathrm{m}$ from the lesion site, with (d) or without any rehabilitation (e) and colocalization with neurons showing PNN structures $(\boldsymbol{f})$. $\boldsymbol{g}$-i, Fluorescence images of aggrecan $200 \mu \mathrm{m}$ from the lesion site, with $(\boldsymbol{g})$ or without any rehabilitation $(\boldsymbol{h})$ and colocalization with neurons showing PNN structures (i). $\boldsymbol{j}, \boldsymbol{k}$, Fluorescence images of ( $\mathbf{r t} 1 \mathbf{1} 200 \mu \mathrm{m}$ from the lesion site after ChABC treatment showing the removal of perineuronal staining (j) and colocalization with neurons showing some upregulation inside the cell $(\boldsymbol{k}) . \boldsymbol{I}, \boldsymbol{m}$, Fluorescence images of WFA $200 \mu \mathrm{m}$ from the lesion site after ChABC treatment showing the removal of WFA staining $(\boldsymbol{I})$ and colocalization with neurons ( $\boldsymbol{m}$ ). Scale bar, $100 \mu \mathrm{m}$. $\boldsymbol{n}$, Quantification of relative intensity of Crtl1, WFA, and aggrecan $200 \mu \mathrm{m}$ from the lesion site with or without rehabilitation. Rehabilitation significantly increased the level of Crtl1 around the lesion (** $p<0.01)$. o, Quantification of the percentage of neurons with Crtl1 ${ }^{+v e}$, WFA ${ }^{+ \text {ve }}$, or aggrecan ${ }^{+ \text {ve }}$ PNNs. Rehabilitation significantly increased the percentage of Crtl1 ${ }^{+}$ve net carrying neurons $\left({ }^{*} p<0.05\right)$. There was an increase in the percentage of neurons with WFA ${ }^{+v e}$ nets with rehabilitation; however, this was not statistically significant. $\boldsymbol{p}$, Quantification of thickness of the $\mathrm{Crtl}^{+\mathrm{ve}}$, WFA ${ }^{+\mathrm{ve}}$, or aggrecan ${ }^{+\mathrm{ve}}$ PNNs. Rehabilitation slightly but significantly increased the thickness of the Crtl1 ${ }^{+\mathrm{ve}}$ nets.

ChABC is to digest PNNs (Bertolotto et al., 1995; Massey et al., 2006; Carulli et al., 2010), which may make it possible for new sprouts to access the surface of neurons, increasing their probability of forming a synapse. It is probable that the initial connections formed by axon sprouts are random, some functionally appropriate, some inappropriate. The situation is therefore similar to the exuberant connections formed during development. Rehabilitation might then instruct the process of refinement of these connections, strengthening appropriate and removing inappropriate connections (Fawcett and Curt, 2009; Kanagal and Muir, 2009). It could also induce cortical reorganization leading to extensive cortical map changes that have been correlated to recovery in rat SCI (Ramanathan et al., 2006; Ghosh et al., 2009). We examined the effects of rehabilitation alone on PNNs, finding an increase in their number and in the levels of Crtl1 and WFA. A link between the amount of activity and PNN formation has been seen in the visual cortex and barrel cortex, and around motorneurons, with reduced PNN formation upon reduced activity (Kalb and Hockfield, 1988; Sur et al., 1988; Guimarães et al., 1990; Kind et al., 1995; Lander et al., 1997; Pizzorusso et al., 2002;
McRae et al., 2007). However, environmental enrichment in amblyopia decreases PNN numbers (Sale et al., 2007).

The formation of PNNs stimulated by upregulation of Crtl1 limits CNS plasticity at the end of critical periods (Sur et al., 1988; Pizzorusso et al., 2002; Carulli et al., 2010), and PNN removal with $\mathrm{ChABC}$ restores plasticity. It therefore seems that rehabilitation may impede its own success by upregulating PNNs. This may partially explain the difficulty of adult rehabilitation after SCI, and emphasizes the importance of ChABC digestion in removing existing PNNs, preventing their upregulation by activity and thereby allowing the changes in circuitry that underlie functional recovery. Delayed rehabilitation in the present study led to some recovery in nonrehabilitated sensorimotor tasks. Whether this improvement is a learned behavioral adaptation or an indication of changes in connectivity is unclear; increased deposition of PNNs may or may not be relevant to this partial recovery. An examination of the relative timings of recovery and of PNN deposition in different behaviors should be revealing.

Our experiment shows that delayed ChABC treatment remains able to induce sprouting of different spinal fibers in 
chronic SCI, and that this increase in sprouting is sufficient to drive functional recovery when combined with rehabilitation. The timing and methodology we used could be applied to human SCI patients.

\section{References}

Allred RP, Jones TA (2008) Maladaptive effects of learning with the lessaffected forelimb after focal cortical infarcts in rats. Exp Neurol 210:172-181.

Barritt AW, Davies M, Marchand F, Hartley R, Grist J, Yip P, McMahon SB, Bradbury EJ (2006) Chondroitinase ABC promotes sprouting of intact and injured spinal systems after spinal cord injury. J Neurosci 26:10856-10867.

Basso DM, Beattie MS, Bresnahan JC (1995) A sensitive and reliable locomotor rating scale for open field testing in rats. J Neurotrauma 12:1-21.

Basso DM, Beattie MS, Bresnahan JC (1996) Graded histological and locomotor outcomes after spinal cord contusion using the NYU weight-drop device versus transection. Exp Neurol 139:244-256.

Bertolotto A, Manzardo E, Iudicello M, Lovisetto C, Riccio A (1995) Disappearance of the Vicia villosa-positivity from the perineuronal net containing chondroitin proteoglycan after chondroitinase digestion. Brain Res 673:344-348.

Bradbury EJ, Moon LD, Popat RJ, King VR, Bennett GS, Patel PN, Fawcett JW, McMahon SB (2002) Chondroitinase ABC promotes functional recovery after spinal cord injury. Nature 416:636-640.

Cafferty WB, Bradbury EJ, Lidierth M, Jones M, Duffy PJ, Pezet S, McMahon SB (2008) Chondroitinase ABC-mediated plasticity of spinal sensory function. J Neurosci 28:11998-12009.

Caggiano AO, Zimber MP, Ganguly A, Blight AR, Gruskin EA (2005) Chondroitinase $\mathrm{ABCI}$ improves locomotion and bladder function following contusion injury of the rat spinal cord. J Neurotrauma 22:226-239.

Carulli D, Pizzorusso T, Kwok JC, Putignano E, Poli A, Forostyak S, Andrews MR, Deepa SS, Glant TT, Fawcett JW (2010) Animals lacking link protein have attenuated perineuronal nets and persistent plasticity. Brain 133:2331-2347.

Cotman CW, Berchtold NC, Christie LA (2007) Exercise builds brain health: key roles of growth factor cascades and inflammation. Trends Neurosci 30:464-472.

Coumans JV, Lin TT, Dai HN, MacArthur L, McAtee M, Nash C, Bregman BS (2001) Axonal regeneration and functional recovery after complete spinal cord transection in rats by delayed treatment with transplants and neurotrophins. J Neurosci 21:9334-9344.

De Leon RD, Hodgson JA, Roy RR, Edgerton VR (1998a) Locomotor capacity attributable to step training versus spontaneous recovery after spinalization in adult cats. J Neurophysiol 79:1329-1340.

De Leon RD, Hodgson JA, Roy RR, Edgerton VR (1998b) Full weight-bearing hindlimb standing following stand training in the adult spinal cat. J Neurophysiol 80:83-91.

Fawcett JW, Curt A (2009) Damage control in the nervous system: rehabilitation in a plastic environment. Nat Med 15:735-736.

Fawcett JW, Curt A, Steeves JD, Coleman WP, Tuszynski MH, Lammertse D, Bartlett PF, Blight AR, Dietz V, Ditunno J, Dobkin BH, Havton LA, Ellaway PH, Fehlings MG, Privat A, Grossman R, Guest JD, Kleitman N, Nakamura M, Gaviria M, Short D (2007) Guidelines for the conduct of clinical trials for spinal cord injury as developed by the ICCP panel: spontaneous recovery after spinal cord injury and statistical power needed for therapeutic clinical trials. Spinal Cord 45:190-205.

Feng SQ, Zhou XF, Rush RA, Ferguson IA (2008) Graft of pre-injured sural nerve promotes regeneration of corticospinal tract and functional recovery in rats with chronic spinal cord injury. Brain Res 1209:40-48.

Fraidakis MJ, Spenger C, Olson L (2004) Partial recovery after treatment of chronic paraplegia in rat. Exp Neurol 188:33-42.

Galtrey CM, Asher RA, Nothias F, Fawcett JW (2007) Promoting plasticity in the spinal cord with chondroitinase improves functional recovery after peripheral nerve repair. Brain 130:926-939.

García-Alías G, Lin R, Akrimi SF, Story D, Bradbury EJ, Fawcett JW (2008) Therapeutic time window for the application of chondroitinase ABC after spinal cord injury. Exp Neurol 210:331-338.

García-Alías G, Barkhuysen S, Buckle M, Fawcett JW (2009) Chondroitinase $\mathrm{ABC}$ treatment opens a window of opportunity for task-specific rehabilitation. Nat Neurosci 12:1145-1151.

Ghosh A, Sydekum E, Haiss F, Peduzzi S, Zörner B, Schneider R, Baltes C,
Rudin M, Weber B, Schwab ME (2009) Functional and anatomical reorganization of the sensory-motor cortex after incomplete spinal cord injury in adult rats. J Neurosci 29:12210-12219.

Girgis J, Merrett D, Kirkland S, Metz GA, Verge V, Fouad K (2007) Reaching training in rats with spinal cord injury promotes plasticity and task specific recovery. Brain 130:2993-3003.

Guimarães A, Zaremba S, Hockfield S (1990) Molecular and morphological changes in the cat lateral geniculate nucleus and visual cortex induced by visual deprivation are revealed by monoclonal antibodies Cat-304 and Cat-301. J Neurosci 10:3014-3024.

Hagg T, Baker KA, Emsley JG, Tetzlaff W (2005) Prolonged local neurotrophin-3 infusion reduces ipsilateral collateral sprouting of spared corticospinal axons in adult rats. Neuroscience 130:875-887.

Hill CE, Beattie MS, Bresnahan JC (2001) Degeneration and sprouting of identified descending supraspinal axons after contusive spinal cord injury in the rat. Exp Neurol 171:153-169.

Houle JD, Tessler A (2003) Repair of chronic spinal cord injury. Exp Neurol 182:247-260.

Hu R, Zhou J, Luo C, Lin J, Wang X, Li X, Bian X, Li Y, Wan Q, Yu Y, Feng H (2010) Glial scar and neuroregeneration: histological, functional, and magnetic resonance imaging analysis in chronic spinal cord injury. J Neurosurg Spine 13:169-180.

Hyatt AJ, Wang D, Kwok JC, Fawcett JW, Martin KR (2010) Controlled release of chondroitinase $\mathrm{ABC}$ from fibrin gel reduces the level of inhibitory glycosaminoglycan chains in lesioned spinal cord. J Control Release 147:24-29.

Iseda T, Okuda T, Kane-Goldsmith N, Mathew M, Ahmed S, Chang YW, Young W, Grumet M (2008) Single, high-dose intraspinal injection of chondroitinase reduces glycosaminoglycans in injured spinal cord and promotes corticospinal axonal regrowth after hemisection but not contusion. J Neurotrauma 25:334-349.

Kadoya K, Tsukada S, Lu P, Coppola G, Geschwind D, Filbin MT, Blesch A, Tuszynski MH (2009) Combined intrinsic and extrinsic neuronal mechanisms facilitate bridging axonal regeneration one year after spinal cord injury. Neuron 64:165-172.

Kalb RG, Hockfield S (1988) Molecular evidence for early activitydependent development of hamster motor neurons. J Neurosci 8:2350-2360.

Kanagal SG, Muir GD (2009) Task-dependent compensation after pyramidal tract and dorsolateral spinal lesions in rats. Exp Neurol 216:193-206.

Karimi-Abdolrezaee S, Eftekharpour E, Wang J, Morshead CM, Fehlings MG (2006) Delayed transplantation of adult neural precursor cells promotes remyelination and functional neurological recovery after spinal cord injury. J Neurosci 26:3377-3389.

Karimi-Abdolrezaee S, Eftekharpour E, Wang J, Schut D, Fehlings MG (2010) Synergistic effects of transplanted adult neural stem/progenitor cells, chondroitinase, and growth factors promote functional repair and plasticity of the chronically injured spinal cord. J Neurosci 30:1657-1676.

Keyvan-Fouladi N, Raisman G, Li Y (2003) Functional repair of the corticospinal tract by delayed transplantation of olfactory ensheathing cells in adult rats. J Neurosci 23:9428-9434.

Keyvan-Fouladi N, Raisman G, Li Y (2005) Delayed repair of corticospinal tract lesions as an assay for the effectiveness of transplantation of Schwann cells. Glia 51:306-311.

Kind PC, Beaver CJ, Mitchell DE (1995) Effects of early periods of monocular deprivation and reverse lid suture on the development of Cat-301 immunoreactivity in the dorsal lateral geniculate nucleus (dLGN) of the cat. J Comp Neurol 359:523-536.

Krajacic A, Weishaupt N, Girgis J, Tetzlaff W, Fouad K (2010) Traininginduced plasticity in rats with cervical spinal cord injury: effects and side effects. Behav Brain Res 214:323-331.

Lammertse D, Tuszynski MH, Steeves JD, Curt A, Fawcett JW, Rask C, Ditunno JF, Fehlings MG, Guest JD, Ellaway PH, Kleitman N, Blight AR, Dobkin BH, Grossman R, Katoh H, Privat A, Kalichman M (2007) Guidelines for the conduct of clinical trials for spinal cord injury as developed by the ICCP panel: clinical trial design. Spinal Cord 45:232-242.

Lander C, Kind P, Maleski M, Hockfield S (1997) A family of activitydependent neuronal cell-surface chondroitin sulfate proteoglycans in cat visual cortex. J Neurosci 17:1928-1939.

Lin R, Kwok JC, Crespo D, Fawcett JW (2008) Chondroitinase ABC has a long lasting effect on chondroitin sulphate glycosaminoglycan content in the injured rat brain. J Neurochem 104:400-408. 
López-Vales R, Forés J, Verdú E, Navarro X (2006) Acute and delayed transplantation of olfactory ensheathing cells promote partial recovery after complete transection of the spinal cord. Neurobiol Dis 21:57-68.

López-Vales R, Forés J, Navarro X, Verdú E (2007) Chronic transplantation of olfactory ensheathing cells promotes partial recovery after complete spinal cord transection in the rat. Glia 55:303-311.

Lu J, Féron F, Mackay-Sim A, Waite PM (2002) Olfactory ensheathing cells promote locomotor recovery after delayed transplantation into transected spinal cord. Brain 125:14-21.

Massey JM, Hubscher CH, Wagoner MR, Decker JA, Amps J, Silver J, Onifer SM (2006) Chondroitinase ABC digestion of the perineuronal net promotes functional collateral sprouting in the cuneate nucleus after cervical spinal cord injury. J Neurosci 26:4406-4414.

McKenna JE, Whishaw IQ (1999) Complete compensation in skilled reaching success with associated impairments in limb synergies, after dorsal column lesion in the rat. J Neurosci 19:1885-1894.

McRae PA, Rocco MM, Kelly G, Brumberg JC, Matthews RT (2007) Sensory deprivation alters aggrecan and perineuronal net expression in the mouse barrel cortex. J Neurosci 27:5405-5413.

Metz GA, Schwab ME (2004) Behavioral characterization in a comprehensive mouse test battery reveals motor and sensory impairments in growthassociated protein-43 null mutant mice. Neuroscience 129:563-574.

Montoya CP, Campbell-Hope LJ, Pemberton KD, Dunnett SB (1991) The "staircase test": a measure of independent forelimb reaching and grasping abilities in rats. J Neurosci Methods 36:219-228.

Moon LD, Asher RA, Rhodes KE, Fawcett JW (2002) Relationship between sprouting axons, proteoglycans and glial cells following unilateral nigrostriatal axotomy in the adult rat. Neuroscience 109:101-117.

Nishio Y, Koda M, Kitajo K, Seto M, Hata K, Taniguchi J, Moriya H, Fujitani M, Kubo T, Yamashita T (2006) Delayed treatment with Rho-kinase inhibitor does not enhance axonal regeneration or functional recovery after spinal cord injury in rats. Exp Neurol 200:392-397.

Pettersson LG, Alstermark B, Blagovechtchenski E, Isa T, Sasaski S (2007) Skilled digit movements in feline and primate-recovery after selective spinal cord lesions. Acta Physiol (Oxf) 189:141-154.

Pizzorusso T, Medini P, Berardi N, Chierzi S, Fawcett JW, Maffei L (2002) Reactivation of ocular dominance plasticity in the adult visual cortex. Science 298:1248-1251.

Ramanathan D, Conner JM, Tuszynski MH (2006) A form of motor cortical plasticity that correlates with recovery of function after brain injury. Proc Natl Acad Sci U S A 103:11370-11375.

Rudge JS, Silver J (1990) Inhibition of neurite outgrowth on astroglial scars in vitro. J Neurosci 10:3594-3603.

Sale A, Maya Vetencourt JF, Medini P, Cenni MC, Baroncelli L, De Pasquale R, Maffei L (2007) Environmental enrichment in adulthood promotes amblyopia recovery through a reduction of intracortical inhibition. Nat Neurosci 10:679-681.

Schwab ME, Bartholdi D (1996) Degeneration and regeneration of axons in the lesioned spinal cord. Physiol Rev 76:319-370.

Shumsky JS, Tobias CA, Tumolo M, Long WD, Giszter SF, Murray M (2003) Delayed transplantation of fibroblasts genetically modified to secrete BDNF and NT-3 into a spinal cord injury site is associated with limited recovery of function. Exp Neurol 184:114-130.

Silver J, Miller JH (2004) Regeneration beyond the glial scar. Nat Rev Neurosci 5:146-156.

Starkey ML, Barritt AW, Yip PK, Davies M, Hamers FP, McMahon SB, Bradbury EJ (2005) Assessing behavioural function following a pyramidotomy lesion of the corticospinal tract in adult mice. Exp Neurol 195:524-539.
Steeves JD, Lammertse D, Curt A, Fawcett JW, Tuszynski MH, Ditunno JF, Ellaway PH, Fehlings MG, Guest JD, Kleitman N, Bartlett PF, Blight AR, Dietz V, Dobkin BH, Grossman R, Short D, Nakamura M, Coleman WP, Gaviria M, Privat A (2007) Guidelines for the conduct of clinical trials for spinal cord injury (SCI) as developed by the ICCP panel: clinical trial outcome measures. Spinal Cord 45:206-221.

Stichel CC, Müller HW (1994) Extensive and long-lasting changes of glial cells following transection of the postcommissural fornix in the adult rat. Glia 10:89-100.

Sur M, Frost DO, Hockfield S (1988) Expression of a surface-associated antigen on Y-cells in the cat lateral geniculate nucleus is regulated by visual experience. J Neurosci 8:874-882.

Tester NJ, Howland DR (2008) Chondroitinase ABC improves basic and skilled locomotion in spinal cord injured cats. Exp Neurol 209:483-496.

Tobias CA, Shumsky JS, Shibata M, Tuszynski MH, Fischer I, Tessler A, Murray M (2003) Delayed grafting of BDNF and NT-3 producing fibroblasts into the injured spinal cord stimulates sprouting, partially rescues axotomized red nucleus neurons from loss and atrophy, and provides limited regeneration. Exp Neurol 184:97-113.

Tom VJ, Sandrow-Feinberg HR, Miller K, Santi L, Connors T, Lemay MA, Houlé JD (2009a) Combining peripheral nerve grafts and chondroitinase promotes functional axonal regeneration in the chronically injured spinal cord. J Neurosci 29:14881-14890.

Tom VJ, Kadakia R, Santi L, Houlé JD (2009b) Administration of chondroitinase $\mathrm{ABC}$ rostral or caudal to a spinal cord injury site promotes anatomical but not functional plasticity. J Neurotrauma 26:2323-2333.

Tuszynski MH, Steeves JD, Fawcett JW, Lammertse D, Kalichman M, Rask C, Curt A, Ditunno JF, Fehlings MG, Guest JD, Ellaway PH, Kleitman N, Bartlett PF, Blight AR, Dietz V, Dobkin BH, Grossman R, Privat A (2007) Guidelines for the conduct of clinical trials for spinal cord injury as developed by the ICCP panel: clinical trial inclusion/exclusion criteria and ethics. Spinal Cord 45:222-231.

Vaynman S, Gomez-Pinilla F (2005) License to run: exercise impacts functional plasticity in the intact and injured central nervous system by using neurotrophins. Neurorehabil Neural Repair 19:283-295.

Whishaw IQ, Pellis SM, Gorny B, Kolb B, Tetzlaff W (1993) Proximal and distal impairments in rat forelimb use in reaching follow unilateral pyramidal tract lesions. Behav Brain Res 56:59-76.

Whishaw IQ, Gorny B, Sarna J (1998) Paw and limb use in skilled and spontaneous reaching after pyramidal tract, red nucleus and combined lesions in the rat: behavioral and anatomical dissociations. Behav Brain Res 93:167-183.

Yick LW, Wu W, So KF, Yip HK, Shum DK (2000) Chondroitinase ABC promotes axonal regeneration of Clarke's neurons after spinal cord injury. Neuroreport 11:1063-1067.

Yick LW, Cheung PT, So KF, Wu W (2003) Axonal regeneration of Clarke's neurons beyond the spinal cord injury scar after treatment with chondroitinase ABC. Exp Neurol 182:160-168.

Ying Z, Roy RR, Zhong H, Zdunowski S, Edgerton VR, Gomez-Pinilla F (2008) BDNF-exercise interactions in the recovery of symmetrical stepping after a cervical hemisection in rats. Neuroscience 155:1070-1078.

Zuo J, Neubauer D, Dyess K, Ferguson TA, Muir D (1998) Degradation of chondroitin sulfate proteoglycan enhances the neurite-promoting potential of spinal cord tissue. Exp Neurol 154:654-662.

Zurita M, Vaquero J, Oya S, Montilla J (2001) Functional recovery in chronic paraplegic rats after cografts of fetal brain and adult peripheral nerve tissue. Surg Neurol 55:249-254. 